\title{
I lineamenti di Dio nella riflessione sapienziale di Qohelet
}

\section{The features of God in the wisdom reflection of Qohelet}

Vincenzo Cuffaro

\begin{abstract}
Ebbene, figurati che, in ultima analisi, questo mondo di Dio non l'accetto, pur sapendo che esiste, anzi non l'ammetto per nulla. Non è Dio che non accetto, comprendi, ma il mondo da Lui creato.
\end{abstract} (Fëdor M. Dostoevskij, I fratelli Karamazov)

\section{Riassunto}

L'articolo si propone di indagare le cause delle contrastanti affermazioni di Qohelet su Dio, le cui contraddizioni hanno indotto gli studiosi di questi ultimi cinquant'anni a formulare giudizi del tutto diversi sul medesimo libro. L'autore è stato pertanto collocato nella categoria dei credenti oppure in quella degli atei; è stato considerato un pessimista o addirittura un nichilista, oppure un amante della vita e delle sue gioie. È stato perfino negato che il Dio di Qohelet sia il medesimo Dio della tradizione giudaica. Queste anomalie non possono rimanere senza un'adeguata spiegazione. L'articolo giunge alla conclusione che le contraddizioni presenti nel testo sono solo apparenti, in quanto risultano da due punti di vista paralleli e contemporanei, che fondano la ricerca empirica di Qohelet: da un lato il dogma, per cui il mondo è retto dall'ordine divino, e dall'altro l'osservazione della vita, che invece racconta una storia di disordini e di ingiustizie. 
Parole chiave: Dio creatore. Retribuzione. Dogma. Libero arbitrio. Osservazione empirica.

\begin{abstract}
The article points out the nature of contradictions which we find often within the thought of Qohelet. In the last fifteen years the scholars have attributed several and different characteristics to the author: some of them think that he is a believer and some other an atheist; others still think he is a pessimist, a nihilist or a person who loves life and its joy. It has been even denied that the Qohelet's God is the same God of Jewish tradition. These anomalies cannot remain without an adequate explanation. The article concludes that the contradictions in the book of Qohelet are merely apparent. Indeed, they are the result of two different points of view parallel and simultaneous in the Qohelet's research: the dogma, which describes the world under a divine order, and empirical observation, which tells a story of disorder and injustice.
\end{abstract}

Keywords: Creator. Retribution. Dogma. Free will. Empirical observation.

\title{
Introduzione
}

La scelta di focalizzare il Dio di Qohelet in questo contributo, nasce dal fatto che una tale tematica appare alquanto controversa. Infatti, il pensiero del saggio palestinese non si sviluppa in linea retta e coerente, né le sue asserzioni sono prive di apparenti contraddizioni. Il lettore rimane, insomma, piuttosto perplesso dinanzi a una prospettiva abbastanza variegata e non omogenea. Ciò si osserva già in rapporto al tema della sapienza: essa è utile e inutile al tempo stesso: conoscere la sapienza e la scienza è come correre dietro al vento (Qo $1,17 ; 2,14-15)$; il dolore aumenta in proporzione del sapere (Qo 1,18); ma il saggio è più forte di dieci uomini potenti e la sapienza è sempre migliore della forza (Qo 7,19; 9,16-18); eppure, la sapienza rimane irraggiungibile anche per chi la cerca con impegno (Qo 7,23-24) e chi dice di conoscerla, s'inganna (Qo 8,17). Ma si osserva anche in rapporto all'immagine di Dio che emerge dall'insieme dell'opera e che ha portato non pochi esegeti a utilizzare quelle che N. Lohfink definisce "etichette stereotipe", quali ad esempio: "nessun Dio personale', 'negazione della libertà umana', 'apostasia dal pensiero 
storico-salvifico". ${ }^{1}$ In sede scientifica le "etichette" e le "catalogazioni" possono risultare utili ai fini di un possibile orientamento della ricerca in un materiale particolarmente complesso, ma raramente esprimono l'intero di una data realtà. Nel caso del pensiero di Qohelet, una tale metodologia certamente non sembra appropriata, in quanto, non di rado, un'asserzione coesiste con quella rispettivamente contraddittoria. E non fa eccezione la teologia del libro: il Dio che emerge dal testo coincide sostanzialmente coi dati dogmatici della tradizione, ma la riflessione dell'autore sulle sue opere storiche sembra distanziarsene. Per questa ragione l'autore è stato accusato, di volta in volta, di essere uno scettico, un agnostico, un cinico, un nichilista. ${ }^{2}$ S. de Jong apre significativamente un suo articolo del $1997^{3}$ citando due estremi del giudizio degli esegeti sulla teologia di Qohelet: da un lato W. Zimmerli, per il quale il Dio di Qohelet è lo stesso Dio della fede d'Israele, ${ }^{4}$ e dall'altro A. Lauha, che afferma il contrario. ${ }^{5}$ La stesura di un articolo sulla teologia di Qohelet implica anche la formulazione di una risposta alla domanda posta da S. De Jong: "The contradictions in Qohelet's speaking about God raise the question if Qohelet aimed at presenting a more or less complete doctrine of God". ${ }^{6}$ La risposta, in parte, proviene direttamente dalla tradizione degli agiografi: nessun autore dei libri biblici ha inteso fornire al lettore una dottrina "completa" su Dio. Si tratta piuttosto di una descrizione delle opere storiche di Dio, oppure della riflessione su alcune di esse. Qohelet cade ovviamente nella seconda categoria. Per un'altra parte, le incongruenze del suo pensiero su Dio, a nostro giudizio, derivano dal duplice binario su cui si muove il maestro: il metodo empirico e la fede nel dogma. Quest'ultimo offre una data visione dell'umanità posta nel mondo, mentre l'osservazione empirica conduce il sapiente a ricostruire una storia diversa. Qohelet considera vere entrambe le prospettive, anche se non coincidono nei loro singoli enunciati. Avremo modo di tornare su questo.

Nostro intendimento, in questa sede, è di ricostruire il pensiero teologico di Qohelet, non con l'intento di armonizzare quanto appare contraddittorio, ma di chiarire i singoli punti che tratteggiano l'immagine di Dio, ricostruibile dai dati forniti dal testo. Il presente articolo risulta così composto da due parti

\footnotetext{
${ }^{1}$ LOHFINK, N., Qohelet, p. 27.

${ }^{2}$ LÍNDEZ, J. V., Qoèlet, p. 26-31.

${ }^{3}$ DE JONG, S., God in the Book of Qohelet, p. 154-67.

${ }^{4}$ RINGGREN, H.; ZIMMERLI, W., Sprüche / Prediger, p. 133.

5 "Sein Gott ist nicht der Gott des israelitischen Glaubens" (LAHUA, A., Kohelet, p. 17).

${ }^{6}$ DE JONG, S., God in the Book of Qohelet, p. 161.
} 
principali: ci chiediamo, innanzitutto, quali siano le opere di Dio considerate da Qohelet e quale immagine della divinità ne emerga. In un secondo momento, cercheremo di delineare come si configuri la relazione del credente con questo Dio, sia nel quadro dell'esistenza umana, sia nella pratica del culto.

\section{Le opere storiche del Dio conosciuto da Qohelet}

Il metodo teologico di Qohelet non differisce sostanzialmente da quello delle altre tradizioni bibliche: non è mai in questione alcuna considerazione sulla natura di Dio, ma soltanto un punto di partenza di carattere empirico, rappresentato dalle opere storiche che la fede giudaica attribuisce al Dio d'Israele. Anche se manca ogni riferimento all'opera esodale di liberazione, nondimeno non si può avere alcun ragionevole dubbio sul fatto che si tratti del medesimo Dio conosciuto fin dalle tradizioni patriarcali. ${ }^{7}$ Se poi nel quadro complessivo potranno emergere delle contraddizioni, esse avranno la loro origine - a nostro giudizio - non da un pensiero incoerente, ma dall'osservazione degli eventi della vita, di cui la contraddizione e il paradosso costituiscono talvolta una parte integrante, mentre il dogma della fede d'Israele vede nel mondo un'entità morale ordinata dal Dio creatore. Volgiamoci adesso ai singoli punti discussi da Qohelet circa le opere storiche di Dio.

\subsection{Il Dio creatore}

Dal complesso delle tradizioni giudaiche, Qohelet riceve un'immagine di Dio come l'architetto del mondo, ossia colui che ne è l'iniziatore. Si tratta piuttosto di vedere come la nozione del Dio creatore sia entrata nella riflessione del maestro. In questo ambito, l'osservazione empirica si combina con il dato derivante dalla fede. Quest'ultima offre la base per intendere i fenomeni della natura e della storia in un orizzonte di senso, offre cioè il postulato del mondo come entità morale. Esso emerge con molta evidenza nella pericope 3,1-8, dove l'annuncio di un tempo in cui "ogni cosa" è destinata ad accadere si

\footnotetext{
${ }^{7}$ Sotto questo profilo siamo nella linea del già citato W. Zimmerli e di R. N. Whybray, il quale afferma: "It is true that Qoheleth makes no direct reference to divine action in history, the so-called Heilsgeschichte, but is also true of the earlier wisdom books of Proverbs and Job" (WHYBRAY, R. N. Qoheleth as a theologian, p. 247). A chi poi obiettasse che il Dio di Qohelet appare troppo lontano e stranamente distaccato dal mondo, si può rispondere osservando che in fondo anche $\mathrm{i}$ Salmi, non di rado, trasmettono una tale idea di Dio, a volte chiamato perfino a risvegliarsi da un sonno di indifferenza (Sal 44,24-25).
} 
connette all'enunciato di Qo 3,11a, che dichiara "bella" (יפה) ogni cosa, in quanto si situa nel "suo" tempo (בעתו). In questa prospettiva dogmatica, anche gli eventi peggiori e meno desiderabili, acquistano un carattere di maggiore accettabilità; perfino la morte diventa "bella", al tempo suo. ${ }^{8}$ Quando poi Qohelet passa dal livello del creduto a quello del vissuto, emergono delle disarmonie, che sono tali solo rispetto alla visione soggettiva del saggio. Infatti, l'opera compiuta da Dio, dall'inizio alla fine, viene sottratta alla comprensione e agli sforzi intellettuali dei sapienti (Qo 3,11cd). Ciò implica che la disarmonia, o la contraddizione avvertita dal lettore, non si trova nelle opere di Dio, a cui accenna l'autore, bensì nel limite umano di comprensione, tracciato da Dio stesso, insieme alle altre leggi immanenti che regolano la vita del cosmo. Le parole conclusive del v. 11 appaiono, infatti, orientate a liberare il lettore dalle perplessità, che potranno sorgere da uno sviluppo dottrinale apparentemente incoerente sotto diversi aspetti: Dio ha messo la nozione dello על nel loro cuore "senza che possa scoprire l'uomo l'opera di Dio, compiuta dall'inizio alla fine". ${ }^{9}$ La nozione posta da Dio nel cuore umano è ciò che spinge il saggio verso la ricerca di una soluzione che non troverà. In altre parole, per una particolare disposizione divina, la capacità umana di porre delle domande ultime è nettamente superiore a quella di trovarne le risposte. ${ }^{10} \mathrm{Il}$ limite imposto alla conoscenza umana ha, insomma,

${ }^{8}$ Ho trattato più dettagliatamente la questione della morte in Qohelet nel mio articolo: CUFFARO, V., Il problema della morte, p. 123-144.

${ }^{9}$ La traduzione italiana è nostra. Ciò vale anche per le successive citazioni.

${ }^{10}$ F. Piotti ha collegato l'incapacità umana "di comprendere globalmente l'opera di Dio" all'albero della conoscenza di Gen 2,9.17 (PIOTTI, F., Percezione del "disordine" e "timore di Dio" in Qohelet (II), p. 110). Quanto al significato di עלם: "Si tratterebbe di un termine che indica una durata di tempo indefinita non verificabile dall'esperienza umana, a differenza di 'et che indica il singolo momento vivibile nel tempo" (PIOTTI, F., Percezione del "disordine" e "timore di Dio" in Qohelet (II), p. 127). Sotto questo profilo, ciò che sfugge all'uomo non è il senso dell'evento che accade qui e ora, ma il legame finalistico che dovrebbe rendere sensato lo sviluppo complessivo della storia, nel suo arco temporale indefinito. Quest'ultimo, purtroppo, non è sotto il controllo dell'osservazione empirica. Questa chiave di lettura ci sembra convincente, mentre non ci sentiamo di accettare la posizione di coloro che traducono עלם con "eternità", in quanto questa scelta suggerisce al lettore un concetto astratto e pertanto inconsueto nel pensiero veterotestamentario (LOHFINK, N., Qohelet, p. 60; SEOW, C. L., Ecclesiastes, p. 158). L'idea di una durata indefinita ci appare invece più adeguata sotto ogni punto di vista (MURPHY, R., Ecclesiastes, p. 29; VONACH, A., Gottes Souvränität anerkennen. Zum verständnis der "Kanonformel", p. 392-393). Quanto al collegamento con Gen 2,9.17, che determinerebbe l'idea del limite dell'intelletto umano, ci appare semplicemente congetturale, dal momento che Qohelet non ha bisogno di ricorrere al dogma per definire i limiti della conoscenza umana, ben visibili ed evidenti nell'esperienza di ciò che accade sotto il sole. Lo farà piuttosto per definire 
come risultato il rispecchiamento mentale disarmonico di una realtà che invece è governata da Dio in modo pienamente sensato. Tale orizzonte di senso è posizionato tuttavia oltre i confini della conoscenza intellettuale. Per Qohelet, quindi, ogni discorso su Dio, elaborato da lui stesso, o da chiunque altro, dovrà soggiacere alla condizione di questa irrimediabile distanza. Il problema, posto qui sul piano teorico, si porrà nuovamente - come vedremo - anche sul piano pratico: la relazione del credente con Dio e la modalità del culto sono destinati a soggiacere alla medesima condizione. Per questo motivo, il concetto del timore di Dio assume tonalità piuttosto gravi, ma non differisce sostanzialmente da quello inteso dalla tradizione sapienziale, come principio della sapienza. Infatti, i giusti e i saggi si trovano nelle mani di Dio (Qo 9,1ab). Il timore di Dio è, insomma, l'elemento di raccordo tra l'uomo e עלם Dio, mentre il tentativo di capire l'opera sua, suscitato dalla nozione di posta nel cuore, non lo è.

Qohelet riceve dal dogma biblico il dato dell'esistenza dell'essere umano come creatura. Sotto questo profilo, relativamente alla prima linea della sua riflessione, cioè quella dogmatica, si possono individuare i due racconti della creazione, riportati in Gen 1-2, come base della sua lettura del fenomeno umano. In essi, Dio fa buona ogni cosa; anzi, completata l'opera, essa è definita addirittura "molto" buona (Gen 1,31). La seconda linea della sua ricerca è invece costituita dal metodo empirico, che lo porta a desumere un modello concreto di esistenza umana improntato alla negazione della creaturalità, ossia la prevaricazione e il disordine. Il dogma e l'osservazione empirica del fenomeno umano si contraddicono, eppure, per Qohelet, sono veri entrambi. Egli non dubita, sul piano dogmatico, che il mondo come creazione sia cosa "molto buona". Lo afferma perfino a chiare lettere: "Dio ha fatto l'uomo retto" (Qo 7,29b). Questo enunciato non appartiene ai risultati dell'osservazione empirica, ma al retaggio della fede d'Israele, condivisa dal maestro. Nessuna delle due cose è, infatti, deducibile dall'esperienza, che Dio cioè abbia creato l'uomo e che lo abbia creato senza storture. Al contrario, è proprio l'esperienza quella fonte di informazioni capace di far vacillare il dogma. Siamo dell'idea che, nella mente del maestro, il dogma rimanga saldo nonostante tutto. L'allegoria della vecchiaia, che chiude la raccolta, dimostra

ciò che sfugge al metodo empirico, citando in combinazione, e in modo indiretto, Gen 2,7 e 3,19: la destinazione dello spirito vitale dopo la morte fisica della persona (Qo 12,7). Ma ciò non è osservabile empiricamente; di conseguenza, in Qo 3,21 si manifesta il ragionevole dubbio di un osservatore esterno del fenomeno della morte, apparentemente uguale per uomini e bestie. 
che la sua aspettativa finale non è oscurata dal dubbio radicale, ma è fondata su una speranza di natura dogmatica: il ritorno dello spirito umano a Dio (Qo 12,7). È evidente che le due soluzioni coesistono in lui: il dogma gli permette di affermare la speranza del ritorno a Dio dopo la morte, aggiungendo la parte mancante al testo di Gen 3,19, dove si annuncia soltanto il ritorno alla terra del corpo fisico. Il dogma biblico enuncia la provenienza da Dio dello spirito vitale (Gen 2,7) e indirettamente postula un suo ritorno a chi lo ha donato.

Dio, dunque, ha creato l'uomo e lo ha creato bene. L'osservazione della vita quotidiana, tuttavia, conduce l'osservatore alla formulazione di un ulteriore enunciato: "ma essi cercano molteplici macchinazioni" (Qo 7,29c). ${ }^{11}$ Un esercizio improprio della libertà può quindi alterare seriamente la sostanziale bontà delle cose. In questo caso, l'apparente contraddizione (Dio ha fatto l'uomo retto / essi cercano macchinazioni) proviene dal confronto tra il dato dogmatico e la concretezza del vivere umano. In altri casi, il dogma e l'osservazione empirica concorrono a formare il medesimo enunciato: "Guarda l'opera di Dio: chi può raddrizzare la sua stortura?" (Qo 7,13). Il riferimento all'opera di Dio allude qui, ancora una volta, al mondo dove si svolge la vita umana. Il dogma fa dire a Qohelet che tutto è opera del creatore. L'osservazione del saggio, tuttavia, vi scorge delle storture. Alcune di esse possono spiegarsi alla luce del cattivo uso della libertà. Altre no. A queste ultime si riferisce senza dubbio l'enunciato di Qo 7,13, che si presenta come una specificazione di quello, più generico ma del medesimo tenore, riportato in Qo 1,15. Sembra che qui ci si trovi dinanzi a un'ulteriore contraddizione nel discorso su Dio: come creatore egli ha fatto l'uomo retto (Qo 7,29b) e bella ogni cosa (Qo 3,11 ); eppure ha fatto anche qualcosa di storto? (Qo 7,13). Va ricordato, in primo luogo, che la stortura è ciò che appare al giudizio umano e si colloca, quindi, non sul versante del dogma, ma su quello dell'osservazione empirica. A questo punto, J. V. Líndez si chiede: "Cos'è che Dio ha incurvato?". ${ }^{2} \mathrm{La}$ domanda sembra anche a noi ineludibile. Il carattere generico dell'enunciato fa pensare a tutto ciò che appare all'osservatore come un elemento di disordine: "in concreto a tutto quello che nella natura e nella vita degli uomini

\footnotetext{
${ }^{11}$ Traduco חשבנות con "macchinazioni", tenendo conto anche del significato materiale del sostantivo, che designa i congegni delle macchine da guerra. L'idea è resa molto bene da R. Murphy con "devices", alludendo a un'opera di alterazione della realtà creata: "human beings have marred the divine handiwork by seeking חשבנות", vale a dire "many devices" (MURPHY, R., Ecclesiastes, p. 74-75).

${ }^{12}$ LÍNDEZ, J. V., Qoèlet, p. 328.
} 
ci sembra ingiusto o inevitabile, ad esempio una grande catastrofe naturale, la morte, il generale procedere ingiusto degli uomini" ${ }^{13} \mathrm{Ci}$ sentiamo di poter complessivamente concordare, a condizione di escludere l'ultima frase dell'enunciato: l'ingiustizia umana non può cadere nella categoria di ciò che Dio "ha fatto storto", perché Qohelet stesso l'ha già esclusa, come s'è visto, in 7,29. Qui può trattarsi solo delle storture che sfuggono alla capacità umana di gestire gli eventi. ${ }^{14}$ Dall'altro lato, il maestro non afferma che ciò che l'uomo giudica storto, lo sia anche per Dio. Egli lascia intendere chiaramente che va ricondotto alla regia di Dio, ogni evento che non cade sotto il controllo umano. Questa idea viene rafforzata dall'enunciato sulla durata dell'opera di Dio, che valica i confini della percezione umana: "riconosco che tutto ciò che Dio compie ha una durata indefinita" (Qo 3,14ac). ${ }^{15}$ Inoltre, le risorse dell'ingegno devono arrendersi dinanzi all'impossibilità di modificare l'opera divina: nulla si può aggiungere e nulla togliere (Qo 3,14de). Possiamo allora sintetizzare così il pensiero del maestro: tutti i fenomeni che sfuggono al controllo umano, e che si desidererebbe non accadessero, appaiono all'osservatore come delle storture. Che siano da attribuirsi al divino governo del mondo è il risultato della sua chiave di lettura dogmatica, mentre la loro presunta stortura emerge solo da un punto di vista soggettivo ed empirico. Infatti, significativamente, il versetto successivo chiarisce molto bene questa prospettiva: "nel giorno favorevole, sii allegro; nel giorno avverso, osserva: Dio ha fatto l'uno e l'altro, perché l'uomo non scopra cosa accadrà dopo di lui"' (Qo 7,14). I tempi di gioia e di dolore sono strettamente legati a motivazioni di carattere soggettivo. Ebbene, vi è un'alternanza divina dei tempi (Qo 3,1-8) che sfugge alla comprensione umana, come sfugge parimenti la conoscenza del futuro. Dio distingue così i destini umani, secondo quanto il dogma suggerisce a Qohelet (Qo 5,18-6,2), ma con un criterio retributivo incomprensibile e nascosto a ogni ricercatore. Dall'altro lato, vi sono pure distinzioni di destini, il cui responsabile non è il divino governo del mondo, per quanto misterioso, ma il cattivo uso della libertà: "ho osservato ancora sotto il sole al posto del giudizio l'empietà, al

\footnotetext{
${ }^{13}$ LÍNDEZ, J. V., Qoèlet, p. 328.

${ }^{14}$ Sotto questo profilo, concordo piuttosto con l'interpretazione di F. Piotti, secondo cui, ciò che Dio ha fatto storto in Qo 7,13 e 1,14-15 è individuabile in sintesi nella mancanza di una logica retributiva, nell'arbitrarietà dell'agire divino, nel pervertimento della giustizia e del diritto, nell'oppressione dei deboli, nell'ambivalenza delle ricchezze, nell'ascesa di gente incapace o senza scrupoli e nell'emarginazione degli uomini più degni (PIOTTI, F., Percezione del "disordine" e "timore di Dio" in Qohelet (II), p. 122-125).

15 Traduco con "durata indefinita" il sintagma הוא יהיה לעולם.
} 
posto della giustizia l'iniquità. Ho detto in cuor mio: il giusto e l'empio li giudicherà Dio" (Qo 3,16-17). In questo caso, Dio non solo non è il responsabile di un tale capovolgimento dell'ordine dei valori, ma ne è addirittura il garante e il giudice, secondo il dogma tradizionale creduto da Qohelet. L'apparente contraddizione non si può comunque eliminare: la giustizia di Dio è oggetto di fede, mentre l'ingiustizia del mondo è oggetto dell'esperienza. ${ }^{16}$ Il vero problema sta nel fatto che la fede e l'osservazione empirica dell'esistenza, narrano due storie diverse. Ciò spinge i credenti verso la fede oscura e i non credenti verso l'ironia. Riteniamo, a questo riguardo, del tutto plausibile la lettura di Gb 9,2-9 proposta da R. Carny sulla descrizione delle opere di Dio: "these are the great things he does. A terrible arbitrariness without any moral and legal basis; earthquake, flood, failure of sun and stars and a terrible human fate: these are the "wonders"". ${ }^{17}$ Questa prospettiva sottolinea chiaramente l'antitesi tra gli enunciati dogmatici della fede e l'esperienza della vita già registrati nel testo poetico di Giobbe. Qohelet affronta precisamente questa tensione, senza risolverla, limitandosi ad accettarla come dato di fatto. ${ }^{18}$

\subsection{Il Dio donatore dei beni e arbitro della vita umana}

Nonostante i paradossi dell'esistenza, il Dio di Qohelet è benefico verso l'umanità ed è anche rimuneratore e giudice. Questa specifica interpretazione

${ }^{16}$ D'ALARIO, V., L'assurdità del male nella teodicea di Qohelet, p. 190.

${ }^{17}$ CARNY, P., Theodicy in the Book of Qohelet, p. 72. L'autore ritiene che, nella narrativa veterotestamentaria, vi siano dei passaggi di contestazione dell'agire di Dio, come ad esempio Es 4,24: Dio cerca di fare morire Mosè, dopo che nei versetti precedenti egli stesso aveva manifestato il suo amore per Israele, scegliendo Mosè come liberatore. L'autore parla, infatti, di "a latent, indirect or directly outspoken contestation of divine righteousness" (CARNY, P., Theodicy in the Book of Qohelet, p. 74). L'esegesi e l'ermeneutica tentano poi di giustificare teologicamente una tale prospettiva apparentemente irrispettosa: "Lastly may be remembered the interpretations and translations of many centuries which had the object of softening offensive passages for the respectful pious reader" (CARNY, P., Theodicy in the Book of Qohelet, p. 76). Infine, quanto al pensiero teologico di Qohelet, emerge l'inevitabile contrasto tra il dogma e la realtà: "his observations and experiences brought him to a skeptical position in the face of confessions of faith which were in contrast to reality". Nella medesima linea si colloca anche M. V. Fox: "These dogmas belong to his inherited world construction. They are a model of reality, an indispensable means of filtering data and interpreting reality" (FOX, M. V., The inner-structure of Qohelet's thought, p. 234).

18 "Qohelet semble abandonner toute recherche de sens transcendental. Il ne propose aucune hypothèse d'intellegibilité globale, il se borne à constater l'insondable mystère de l'universe créé” (GIRE, P., Qohelet, p. 50). 
dell'agire di Dio proviene, com'è ovvio, dal dogma tradizionale. Che sia un dono di Dio la possibilità di essere felici, e di fruire di un certo benessere nei propri giorni, non è un fatto deducibile da alcuna esperienza. ${ }^{19}$ Più precisamente, l'esperienza consiste nell'essere felici, mentre l'attribuzione a Dio di tale condizione è un puro e semplice atto di fede. Da qui scaturisce una doppia serie di enunciati, in reciproco contrasto. Ciò che il maestro sa del Dio d'Israele, fa parte del suo credo. Ciò che egli osserva come ricercatore sperimentale, è un'altra storia. Cerchiamo adesso di ricostruire questi due versanti, che strutturano il suo pensiero su Dio.

Egli sostiene che la possibilità di essere felici sia un dono di Dio: "Non c'è un bene maggiore per l'uomo che mangiare e bere e dare a se stesso il frutto delle proprie fatiche. Ho visto che anche questo viene dalle mani di Dio. Infatti, chi può mangiare e vivere nell'impegno, ${ }^{20}$ se non io?"21 (Qo $2,24-25 ; 3,13 ; 5,18)$. Che venga dalle mani di Dio la possibilità di godere del frutto del proprio lavoro, è ovviamente un atto di fede. Sotto questa ottica va, quindi, inteso il verbo di percezione che fonda l'enunciato: "ho visto (ראיתי)". Ciò che Qohelet ha visto non è l'origine della felicità umana, ma la propria felicità (ed eventualmente quella di altri), che la fede d'Israele gli fa attribuire a Dio. Parimenti, nel medesimo contesto, anche l'infelicità è attribuita a un decreto di remunerazione stabilito ancora una volta da Dio: "in concreto, all'uomo che gli è gradito, Dio concede sapienza, scienza e gioia;

${ }^{19}$ Osserva bene, a questo proposito, O. Kaiser: "Glück war in Kohelets Augen nie allein das Ergebnis menschlichen Verhaltens und Handelns, sondern es blieb eine freie Gabe Gottes" (KAISER, O., Die Botschaft des Buches Kohelet, p. 66).

${ }^{20}$ Traduco con "essere felice" l'enigmatico verbo חוש, che letteralmente significa "affrettarsi", ma sembrerebbe fuori contesto. Dietro di esso vi è un dibattito filologico che non ha portato ancora ad alcuna soluzione certa. La versione dei LXX presuppone invece la presenza di un altro verbo, che in greco viene tradotto con $\pi$ í $\tau \alpha$, mantenendo così la simmetria dei verbi "mangiare" e "bere" che figura nel v. 24. Infine, il verbo חוש ha ulteriori significati nell'ebraico mishnaico, potendosi tradurre con "considerare" o "preoccuparsi" (KRÜGER, T., Qohelet, p. 59). Questo significato ci convince di più e ci sembra abbastanza coerente con il contesto: si tratta di realizzare dei progetti mediante la fatica quotidiana, impegnando tutte le proprie risorse.

${ }^{21}$ Il TM ha letteralmente חוץ ממני. Si tratta di un'espressione unica nella Bibbia ma frequente nella Mishna, nel senso appunto di "all'infuori”, "eccetto che” (MURPHY, R., Ecclesiastes, p. 25). Qualcuno preferisce correggere il TM trasformando ממנו in ממני così da riferire a Dio il suffisso, ottenendo l'idea di una gioia che non è fruibile senza di lui (LÍNDEZ, J. V., Qoèlet, p. 221-222; SEOW, C. L., Ecclesiastes, p. 118. 141). La nostra scelta è quella di non emendare il TM e tradurlo come esso si presenta. Di conseguenza, il senso più coerente della traduzione letterale: "chi può mangiare e vivere nell'impegno, se non io?", va ricercato nel ruolo dell'autore fittizio, il re di Israele, a cui Dio ha concesso ricchezze e beni senza numero. 
al peccatore dà l'occupazione di raccogliere e accumulare per chi è gradito a Dio. ${ }^{22} \mathrm{Ma}$ anche questo è vanità e un pascolare il vento" (Qo 2,26; 6,2). Chi non vedrebbe dietro questa diversità di destini, la medesima tesi sostenuta dai redattori delle sezioni di epoca monarchica del libro dei Proverbi, nonché dagli amici di Giobbe? L'osservazione della vita smentisce questo principio retributivo, e Qohelet ne è drammaticamente consapevole, come si vede dalla conclusione del v. 26; nondimeno, tale principio è il postulato necessario della fede nel Dio creatore e giusto giudice, nel quale egli crede: "Ho detto in cuor mio: Dio giudicherà il giusto e l'empio" (Qo 3,17ac). Ma il divino giudizio esula radicalmente dall'osservazione del saggio. Anzi, l'esperienza della vita dimostra piuttosto che "dove non si attua una sentenza, l'opera malvagia è rapidamente compiuta. Per questa ragione, si accresce nel cuore umano il desiderio di agire empiamente"23 (Qo 8,11). La fede nel giusto giudizio di Dio viene, di conseguenza, inquadrata nell'orizzonte del futuro in senso assoluto, ossia senza alcuna precisazione circostanziale: ישפט האלהים (Qo 3,17c).. Un'affermazione di questo genere ritorna nell'esortazione rivolta al giovane, senza precisazioni temporali: "Sii lieto, o giovane, nella tua giovinezza; il tuo cuore si rallegri nei giorni dell'età giovanile. Imbocca le vie del tuo cuore $\mathrm{e}$ dei desideri dei tuoi occhi, ma sappi che, su tutto questo, Dio ti convocherà in giudizio" (Qo 11,9). In ogni caso, si tratta di un futuro indefinito, nel quale Dio si calerà nel ruolo di giudice della vita umana. Ciò ci fa pensare a una qualche forma di speranza escatologica, sebbene dogmaticamente grezza. Torneremo, però, su questo in un paragrafo a parte.

\footnotetext{
${ }^{22}$ Alla domanda sull'identità dell'uomo gradito a Dio si può rispondere alla luce del substrato tradizionale del pensiero di Qohelet: si tratta indubbiamente dell'uomo che coltiva il timore di Dio come virtù basilare. Ma c'è anche un'indicazione concreta contenuta in Qo 9,7: "Su, mangia con gioia il tuo pane e bevi con cuore allegro il tuo vino perché Dio ha già gradito le tue opere". Nella prospettiva di Qohelet, Dio dona questa gioia quotidiana a chi gli è gradito. Concordiamo perciò con l'osservazione di M. Maussion: “On peut d'abord constater que les plaisirs fastueux du roi ne procurent pas la même qualité de joie que celle issue de plaisirs quotidiens donnés par Dieu et que Qohélet énumère à plusieurs reprises dans son œuvre" (MAUSSION, M., Qohélet VI 1-2, p. 506). E in definitiva: "l'homme qui plaît à Dieu serait alors celui qui sait accueillir les joies simples de la vie" (MAUSSION, M., Qohélet VI 1-2, p. 509).

${ }^{23} \mathrm{Il}$ TM potrebbe qui essere tradotto anche in un altro modo: "Poiché non si dà una sentenza rapida su un'opera malvagia, si accresce nel cuore umano il desiderio di agire empiamente" (MURPHY, R., Ecclesiastes, p. 79).

${ }^{24}$ Sotto questo aspetto, osserva opportunamente G. Di Palma: "il Qohelet non può affermare nulla sul quando e sul dove di tale giudizio divino, né può precisarne le caratteristiche. Ma è indubitabile che egli creda a un giudizio di Dio qualitativamente non paragonabile con quello umano" (DI PALMA, G., Il giudizio di Dio nel libro di Qohelet, p. 356).
} 
Le cose dette fin qui, dimostrano ampiamente che la riflessione di Qohelet su Dio si sviluppa dunque su due versanti contemporanei: mentre la fede biblica descrive un mondo retto dall'ordine divino: "So tuttavia che andrà bene a coloro che temono Dio" (Qo 8,12ce; cfr. 7,18), l'osservazione empirica narra la storia della divina assenza: "il peccatore anche se commette il male mille volte ha lunga vita" (Qo 8,12ab). ${ }^{25}$ Inoltre, Dio appare assente laddove ci sono le ingiustizie e il pianto degli oppressi senza consolazione (Qo 4,1). Che il timore di Dio garantisca la sicurezza del vivere all'uomo sapiente, fa parte del creduto; l'osservazione empirica costringe, invece, costatare che "sulla terra avviene un fenomeno vano: vi sono dei giusti trattati come se avessero compiuto le opere dei malvagi e malvagi trattati come se avessero agito da giusti. Ho concluso che questa è una cosa vana" (Qo 8,14). Una tale conclusione non comporta, da parte del maestro, alcuna negazione della fede giudaica, ma solo la presa d'atto di due grandezze che non convergono mai: il dogma e la realtà storica. Ad ogni modo, questo fatto non ci deve meravigliare, dal momento che l'intero panorama della fede cristiana è caratterizzato dal medesimo, inconciliabile doppio binario, nel quale la fede è smentita dalla storia. L'assenza di Dio, a cui allude Qohelet, non è un'affermazione di principio, ma solo ciò che si osserva nel mondo dei fenomeni, ossia quel campo di indagine che egli delimita al di sotto della volta celeste (Qo 3,1). Rimane tuttavia aperto uno scenario, a cui la ricerca del saggio non giunge e dove si collocano le ragioni profonde delle cose; esse sono, infatti, orchestrate da una divina regia situata fuori dal controllo umano. ${ }^{26}$ Del resto, questa prospettiva è già interamente contenuta nel duplice

${ }^{25}$ L. Mazzinghi nota che, nella visuale di Qohelet, il dato empirico dell'assenza di Dio è connesso
alle ingiustizie sociali, citate in Qo 4,1, e in particolare il senso di abbandono degli oppressi, che
non hanno chi li consoli. A suo giudizio, l'autore entra in polemica con Is 40,1, dove Dio stesso
invia a Israele i suoi consolatori (MAZZINGHI, L., The Divine Violence in the Book of Qoheleth,
p. 548 ). Noi non ci sentiamo di accettare una tale conclusione: Qohelet non entra in polemica con
ciò che fa parte integrante della sua fede e che egli aveva già chiaramente espresso, affermando
che Dio concede a chi gli è gradito la possibilità di godere delle gioie semplici della vita (Qo
2,24). Ciò implica che Is 40,1 non manchi di compiersi per il pio israelita. Una tale affermazione,
però, deriva dalla fede e non dall'osservazione empirica della vita, la quale narra invece una storia
di dolori e di ingiustizie. Concordo piuttosto con l'esegeta fiorentino in una sua affermazione
successiva, relativa al medesimo argomento, quando osserva cioè che Qohelet non accusa Dio
per la sua assenza, bensì osserva un fenomeno che cade sotto l'esperienza comune: la realtà della
violenza nel mondo e l'apparente assenza del divino giudizio (MAZZINGHI, L., The Divine
Violence in the Book of Qoheleth, p. 549 ). Si tratta infatti, a nostro modo di vedere, di due diverse
grandezze contemporanee e irriducibili, nelle quali si articola il pensiero del maestro: il dogma
creduto e la realtà osservata. ${ }^{26}$ V. D'Alario ha messo in evidenza questo aspetto del pensiero teologico di Qohelet: 
discorso che Dio rivolge a Giobbe, tra le raffiche dell'uragano (Gb 38,1-41,26): l'ordine e il caos sono destinati a coesistere per un misterioso decreto divino. La vita si svolge sulla terra secondo un ordine prestabilito: l'alternanza delle stagioni, i fenomeni atmosferici e il ciclo vitale degli esseri viventi. Ma i mostri del caos originario non sono stati eliminati: Leviatan e Behemot sono lì a minacciare perennemente l'ordine del creato. Tuttavia, il conflitto tra l'ordine e il caos non genera squilibri distruttivi. Esso viene piuttosto regolamentato da un criterio superiore, cosmico, non identificato dalla ricerca empirica. Anche Qohelet si mostra di questa opinione nel suo pensiero teologico: l'ordine e il caos coesistono sotto un'incomprensibile divina regia. Il saggio osserva queste due grandezze come se fossero separate e indipendenti l'una dall'altra; per questa ragione separatamente le studia e le confronta. In realtà, oltre i confini dell'indagine umana, esse costituiscono insieme un'armonia incomprensibile ma estesa a tutti gli ambiti dell'universo. ${ }^{27}$

A questo riguardo, non possiamo non citare la posizione di M. V. Fox, per il quale, le apparenti contraddizioni del pensiero di Qohelet, dipendono pure dal conflitto tra due grandezze: la prima è appunto l'osservazione della vita - e su questo tutti siamo d'accordo -; la seconda è l'analisi razionale degli eventi, ${ }^{28}$ portata avanti con categorie logiche:

Significance cannot be read out of events. And since the sages set their hearts on understanding and teaching the meaning of behaviour and events, the senslessness of phenomena was, to the wise Qohelet, almost a personal insult. Qohelet sees absurdities in a variety of phenomena belonging to three domains: work and pleasure, wisdom and righteousness. ${ }^{29}$

\footnotetext{
"Più che teorizzare l'assenza di Dio, Qohelet si fa sostenitore della teologia del mistero e dell'indecifrabilità del volere divino. L'assurdità del male è una dimostrazione dell'incapacità dell'uomo di decifrare la volontà di Dio" (D'ALARIO, V., L'assurdità del male nella teodicea di Qohelet, p. 197).

${ }^{27} \mathrm{Su}$ questo non abbiamo bisogno di insistere. S. De Jong ha stilato una lista delle affermazioni di Qohelet su Dio, annotando tra l'altro: egli è creatore e datore di ogni cosa; la sua opera è immutabile; egli determina i destini dei singoli e dei popoli, ma è impossibile individuare $\mathrm{i}$ criteri del suo governo universale (DE JONG, S., God in the Book of Qohelet, p.155-156).

${ }^{28}$ M. V. Fox suggerisce di tradurre הבל con "absurd" e inquadra il suo significato nelle categorie della logica: "The absurd is a disjunction between two phenomena that are thought to be linked by a bond of harmony or causality, or that should be so linked. Such bonds are the sine qua non of rationality, and all deduction and explanation presuppose them" (FOX, M. V., The innerstructure of Qohelet's thought, p. 227).

${ }^{29}$ FOX, M. V., The inner-structure of Qohelet's thought, p. 227.
} 
Per questo egli propone di tradurre il lessema הבל con "assurdità", in quanto si contrappone alle aspettative della ragione. Ad ogni modo, ciò che ci lascia perplessi è il fatto che Fox ponga in antitesi due cose che invece, per un sapiente d'Israele, si trovano sullo stesso piano: l'osservazione esperienziale e l'analisi razionale dei fenomeni. Il loro metodo empirico è un'analisi razionale della realtà. Ciò che contrasta con la realtà è, invece, la parola della fede. Di conseguenza, i termini dell'antitesi stabiliti da Fox non ci sembrano totalmente idonei a spiegare l'atteggiamento del maestro verso i paradossi della vita. Ciò che egli definisce "the senslessness of phenomena", non può essere il risultato di un procedimento razionale. Se così fosse, non ci spiegheremmo per quale ragione i filosofi greci, che hanno interpretato il mondo "razionalmente", non abbiano mai affermato che i fenomeni storici o cosmici siano privi di senso. Al contrario, li hanno giudicati del tutto intelligibili. L'analisi razionale non conduce, dunque, alla formulazione di un tale giudizio. In definitiva, se possiamo concordare con Fox relativamente al metodo di osservazione empirica, applicato da Qohelet alla propria ricerca, non possiamo tuttavia accettare l'idea che egli percepisca il mondo nella categoria dell'assurdo per il fatto di interpretarlo "razionalmente". Diremmo piuttosto che l'insensatezza individuata dal maestro nei fenomeni osservabili sotto il cielo, non sia da addebitarsi al suo "razionalismo", bensì alla sua fede nel Dio creatore e, di conseguenza, al presupposto dogmatico di un mondo concepito come un'entità morale. Proprio da questo conflitto tra l'ordine creduto e il fenomeno storico osservato si origina una visione delle cose debitrice contemporaneamente a entrambe le prospettive, e per questo apparentemente contraddittoria.

\section{L'uomo davanti al mistero di Dio}

\subsection{Le difficoltà del saggio}

La riflessione di Qohelet su Dio, come risulta dalle osservazioni fatte fin qui, si snoda sui presupposti delle fede di Israele. ${ }^{30}$ Il Dio di Qohelet è sostanzialmente il Dio della rivelazione giudaica, i cui dogmi vengono integrati dal saggio nella sua riflessione sul mondo e sulla storia. Rimane ancora un secondo ambito da scandagliare: l'aspetto etico che consegue, o corrisponde, ai dati basilari della teologia presentata dal maestro. La posizione e il destino dei

\footnotetext{
${ }^{30} \mathrm{R}$. N. Whybray giunge alla medesima conclusione nel suo contributo: WHYBRAY, R. N., Qoheleth as a theologian, p. 239-265.
} 
credenti vengono da lui considerati come parte ineliminabile di un discorso su Dio. Anzi, probabilmente è l'unica parte accessibile di un tema, la cui trattazione sfugge a tutti. Non sono raggiungibili i criteri con cui Dio governa l'universo (Qo 3,11), ma sono alla portata del saggio le conseguenze e le implicanze del rapporto religioso con lui (Qo 4,17-5,6). È possibile, quindi, elaborare a questo riguardo degli orientamenti sapienziali. Vediamo in che modo.

Qohelet considera, in primo luogo, la situazione incresciosa del saggio, a cui Dio ha dato il compito di indagare la realtà delle cose, senza tuttavia poter raggiungere alcuna certezza (Qo 3,11). Più precisamente, l'uomo "cannot grasp and comprehend in every individual case how and in what sense that which God made is 'beautiful"'. ${ }^{31}$ Indubbiamente, il problema sta qui: se il dogma del Dio creatore esige che i fenomeni del mondo siano ben ordinati, ovvero "belli", non può che essere così. Di conseguenza, è l'osservatore a trovarsi nell'incapacità di cogliere la bellezza dell'opera di Dio, ma essa è intrinsecamente tale. Stando così le cose, l'attività del saggio si risolve in un madornale fallimento, rispetto alla possibilità di rispondere alle domande finali (Qo 7,23-24). Non lo è, invece, rispetto alle domande penultime: "è un bene la sapienza insieme all'eredità: è un vantaggio per quelli che vedono il sole. Infatti, la protezione della saggezza è come la protezione del denaro. Il risultato della saggezza custodisce in vita il suo possessore" (Qo 7,11-12). Ancora più radicale appare l'affermazione secondo cui la saggezza rafforza il saggio più di dieci governatori nella città (Qo 7,19). In altre parole, la saggezza non è svuotata dai suoi significati o dal suo valore; è solo ridimensionata rispetto alle sue antiche pretese (Qo 8,16-17). Del resto, anche il libro di Giobbe compie un medesimo ridimensionamento: i suoi amici vengono smentiti nella loro convinzione di conoscere troppo bene l'agire di Dio con gli uomini; ma anche Giobbe viene smentito nella sua convinzione che sia possibile discutere con Dio sul tema della giustizia. Per questa ragione, il lavoro del saggio diventa penoso: una fatica quotidiana, spesa in un'attività incessante di ricerca, che risulta utile solo per la soluzione di questioni non definitive (Qo 1,12-14).

\subsection{Il timore di Dio e il libero arbitrio}

La prospettiva etica di Qohelet, a nostro avviso, ruota intorno a due concetti fondamentali, tratti dalla tradizione sapienziale: il timore di Dio e il

\footnotetext{
${ }^{31}$ KRÜGER, T., Qoheleth, p. 87.
} 
libero arbitrio. ${ }^{32}$ Essi sono inseparabili, in quanto l'atteggiamento religioso, che va sotto il nome di "timore", è sostanzialmente il risultato di una libera opzione. Infatti, un tale atteggiamento è possibile solo dinanzi a un'azione divina e a causa di essa. ${ }^{33}$ Una volta posta, essa crea un bivio, nel quale il timore di Dio rappresenta una delle due vie. L'altra, ovviamente, è l'empietà, ossia il rifiuto di accettare Dio in quanto Dio e se stessi in quanto creature. Il maestro applica a sé questa dinamica: "ho compreso che ciò che Dio fa è definitivo e non è possibile aggiungervi o togliervi alcunché. Egli agisce così perché si abbia timore di lui" (Qo 3,14). ${ }^{34}$ Prima del timore religioso vi è dunque un'opera di Dio. Essa sta davanti a ogni persona umana come l'universo sta davanti a Giobbe durante la teofania (Gb 38,1-40,5), cioè come qualcosa di più grande. Qualcosa che valica i confini del suo orizzonte mentale (לעולם). E proprio in questo punto le vie si biforcano. Qohelet sceglie per sé la via del timore, indicandola anche ai suoi discepoli (Qo 5,6). Nella tradizione sapienziale essa è, infatti, concepita come il fondamento della sapienza. La sua prospettiva, sotto questo profilo, si situa nella linea più antica dei maestri d'Israele: "È bene che tu mantenga saldamente questo e non lasci andare quello. Chi teme Dio eviterà entrambi" (Qo 7,18). L'enunciato conclusivo, secondo cui chi teme Dio si salva dai pericoli rappresentati da ciò che è estremo, riflette la visione ottimistica dei saggi della prima generazione. Meno chiara, però, appare l'esortazione alla moderazione, nel momento in cui si analizzano i due versetti precedenti (vv. 16-17). I quattro estremi da evitare sarebbero dunque l'eccessiva giustizia (צריק הרבה), l'eccessiva saggezza (תחרע) (סחכם), l'eccessiva malvagità (תשרע הרבה) e l'essere stolto (סכל). Che la stoltezza sia da evitarsi, non è una questione che possa suscitare molte perplessità.

\footnotetext{
${ }^{32}$ A questo proposito, in riferimento a Qo 3,14, A. Vonach osserva: "Somit besagt v. 14gh (der Gott hat bewirkt, daß man ihn fürchten kann) das Gott selbst es ist, der die Gottesfurcht ermöglicht. Der Mensch wird nicht gezwungen, sondern sie wird ihm von Gott her als Antwort auf dessen Tun angeboten. Ob der Mensch sich dieser Möglichkeit bedient, liegt in seiner eigenen Entscheidungsfreiheit" (VONACH, A., Gottes Souvränität anerkennen. Zum verständnis der "Kanonformel”, p. 395).

${ }^{33}$ R. Murphy ha colto con lucidità questo particolare: "The fact that a verbal form with preposition is used here suggests that Qoheleth is placing greater emphasis upon the action of fearing, on the proper attitude which God wants to evoke in creatures by means of his mysterious action" (MURPHY, R., Ecclesiastes, p. 35).

34 Traduco לעולם con "definitivo" seguendo la scelta ottima di T. Krüger: "I understood that whatever the Deity does is definitive" (KRÜGER, T., Qohelet, p. 80). Le traduzioni che utilizzano termini come "eterno" o "per sempre" non ci convincono, a motivo del loro carattere piuttosto astratto. L'idea da rendere è quella di una durata che supera i limiti della percezione umana.
} 
Affermare, però, che vadano evitate anche le manifestazioni eccessive della giustizia, della saggezza e della malvagità, esige una qualche spiegazione. Si può comprendere anche l'inconveniente di un'eccessiva giustizia, che è il legalismo. Ma difficilmente si potrebbe immaginare un aspetto negativo di una sapienza estrema. Secondo la logica più semplice ed empirica, è infatti meglio essere sapientissimi che sapienti. Ma soprattutto occorre spiegare l'enunciato sulla malvagità, in cui l'enigma potrebbe essere posto in questi termini: se va evitata la malvagità eccessiva, potrebbe ipotizzarsi il fatto che una malvagità misurata possa essere buona, come è senza dubbio buona una giustizia misurata e non eccessiva? L'esortazione conclusiva prevede l'osservanza di entrambe le cose. Giustamente T. Krüger definisce questo versetto "ambiguously formulated" 35 e aggiunge: "the result is the recommendation sometimes to be righteous and sometimes to be unrighteous - according to which one at the moment promises the greatest usefulness". ${ }^{36}$ La prospettiva utilitaristica, a nostro giudizio, sembra la chiave migliore di interpretazione del testo. Non si tratta di un principio generale per un'etica del giusto mezzo, ma di una constatazione dei limiti intrinseci ai vizi e alle virtù. In sostanza, l'agire dei malvagi non conduce ad alcun risultato, ma neppure l'agire dei saggi, in un mondo posto sotto il segno del soffio (הבל), può sperare obiettivi migliori. Qohelet vuole, in sostanza, demolire la convinzione che più si sia giusti e saggi, più si ottengano apprezzabili successi, o - altrimenti detto -, che per vivere meglio, sia necessario essere più giusti e più saggi. Tutto ciò è solo un equivoco. Ma il maestro non lascia sospeso un argomento così grave. Cosa sia necessario per vivere meglio, deve essere scoperto e insegnato. E a questo riguardo, egli attinge alla proposta della tradizione sapienziale: vivere nel timore di Dio (Qo 7,18b). Meno plausibile ci sembra la scelta di intendere l'eccessiva saggezza come un'alterazione negativa, nella linea dell'ipocrisia, suggerita dal verbo חכם costruito all'hitpael. ${ }^{37}$ C.-L. Seow, dal canto suo, nega del tutto che una tale costruzione possa avere il senso grammaticale di una

\footnotetext{
${ }^{35}$ KRÜGER, T., Qohelet, p. 141.

${ }^{36}$ KRÜGER, T., Qohelet, p. 141.

${ }^{37}$ WHYBRAY, R. N., Qoheleth the Immortalist?, p. 191-204. R. Murphy riprende la questione ma osserva: "the whole context of chap. 7 is against the notion that Qoheleth is simply defending traditional wisdom and warning against pretense" (MURPHY, R., Ecclesiastes, p. 70). In questa medesima linea si colloca anche T. Longman III; dopo avere osservato che la forma hitpael non è usata nella prima parte del v. $16 \mathrm{e}$ che vi è una similarità sintattica con il v. 17, conclude: "In this verse, pretense is out of question" (LONGMAN III, T., The Book of Ecclesiastes, p. 195).
} 
manifestazione di falsa sapienza. ${ }^{38}$ Semmai, potrebbe trattarsi del rischio di un'eccessiva sicurezza di sé, ma non di una mancanza di sincerità. ${ }^{39}$

L'approdo significativo è, però, rappresentato dall'invito a vivere nel timore di Dio, dal momento che né la malvagità né la virtù possono garantire nulla, mentre la stoltezza è esclusa senza eccezioni e senza considerazione di misura (Qo 7,17). La malvagità, in certe occasioni e secondo una certa misura, è indubbiamente ammessa da Qohelet ${ }^{40}$ in vista di obiettivi pratici, sebbene non precisati ma lasciati all'intuizione del lettore. In ogni caso, l'esperienza della vita rende ciascuno consapevole del fatto che l'eccessiva dimostrazione di bontà e di mansuetudine, espone una persona alla frode $\mathrm{o}$ alla prevaricazione, più di quanto non accada a chi si mostra duro. Riteniamo che sia questo l'insegnamento pratico che il maestro abbia inteso lasciare ai suoi discepoli, esortandoli indirettamente a osservare un "reasonable' level of wickedness"; ${ }^{41}$ nella società umana, che Qohelet vede piena di ingiustizie, è infatti necessario l'apprendimento di una giusta durezza come atto di custodia e di autodifesa. Dall'altro lato, l'eccesso di sapienza e di giustizia vanno evitati, per non cadere nell'illusione che l'una e l'altra possano garantire risultati positivi o nuovi equilibri per la società. Il timore di Dio invece non inganna ed è in grado di suggerire, nelle singole situazioni, se si debba essere giusti o sapienti, oppure misuratamente rigidi e severi; e fino a che punto (Qo 7,18). Mai suggerirà, ovviamente, di essere stolti.

Il timore di Dio è inoltre sorgente di un complessivo benessere: "il peccatore opera il male cento volte e gli si allunga la vita, sebbene io so che andrà bene a coloro che temono Dio, proprio perché hanno timore al suo cospetto" (Qo 8,12). Questo enunciato ci permette di dimostrare, ancora una volta, il doppio binario dello sviluppo argomentativo del maestro: l'osservazione empirica e il dogma. Ciò che sta sotto i suoi occhi è un'apparente assenza di retribuzione: chi agisce male, lo fa ripetutamente e impunemente. Mentre c'è qualcosa che egli sa (יודע אני) a un diverso livello di conoscenza, anch'essa in qualche modo esperienziale e quindi definibile mediante il verbo ידעי ${ }^{42} \mathrm{Si}$

\footnotetext{
38 "But in classical Hebrew, the verb in this particular pattern (the Hithpael) is never used of pretense" (MURPHY, R., Ecclesiastes, p. 267).

${ }^{39}$ MURPHY, R., Ecclesiastes, p. 267.

${ }^{40}$ Concordo pienamente in questo con T. Longman III: "he warns against excessive wickedness, which leaves open the possibility of a "reasonable" level of wickedness" (LONGMAN III, T., The Book of Ecclesiastes, p. 196).

${ }^{41}$ LONGMAN III, T., The Book of Ecclesiastes, p. 196.

42 È stato notato opportunamente che il verbo ידע è usato qui al participio e non al perfetto.
} 
tratta, concretamente, della conoscenza della fede; come tale, essa non tocca solo la sfera cognitiva ma coinvolge anche la storia personale. In questo secondo livello, egli riconosce come vero, ciò che tuttavia viene smentito dalla quotidianità: ${ }^{43}$ Dio distingue davvero i destini umani, non in base alla giustizia intesa come osservanza della norma, bensì come disposizione d'animo, improntata al timore divino. Sotto questo profilo, per essere gradito a Dio, il giusto deve mutarsi nella figura del sapiente, ridimensionando il valore salvifico della Torah, in quanto codice legislativo. ${ }^{44}$ Nondimeno, entrambi sono nelle mani di Dio (Qo 9,1) ma ciò si conosce e si accoglie come vero grazie al dogma, non certo per via sperimentale.

\subsubsection{Il culto e il timore Dio}

Il timore di Dio si realizza innanzitutto a livello etico e le sue possibili manifestazioni sono state sintetizzate da F. Festorazzi in questi termini: "paura di un Dio incomprensibile"; "riconoscimento della sua bontà". A queste prime due caratteristiche, l'autore connette dei versetti che verbalizzano tali idee. Poi ne aggiunge una terza, senza tuttavia connetterla a un particolare testo; si tratta della "esigenza di rapportarsi a un Dio diverso da quello tradizionale". ${ }^{45}$ Concordiamo in linea di massima con questa sintesi, a esclusione di quest'ultimo enunciato, che l'autore ha preferito non sviluppare in quella sede, lasciandolo in una forma piuttosto generica. Per quel che riguarda il Dio di Qohelet, comunque, noi siamo dell'idea che sia sostanzialmente quello tradizionale. Per essere più precisi, ciò che il

Quest'ultima forma esprime infatti l'oggetto della conoscenza empirica e individuale, mentre la forma participiale esprime una conoscenza pubblica e tradizionale (SEOW, C. L., Ecclesiastes, p. 288).

${ }^{43}$ Molto lucidamente R. Murphy afferma: "Qoheleth knows the teaching about those who fear God, but reality does not square with it” (MURPHY, R., Ecclesiastes, p. 85).

${ }^{44} \mathrm{C}$ 'è infatti una distinzione reale tra il giusto e chi teme Dio. L'elemento distintivo, secondo G. Di Palma - che a sua volta riprende un'idea di P. Sacchi - consiste nel fatto che "il giusto cerca la salvezza mediante l'osservanza della Legge, mentre colui che teme Dio è il sapiente che sa bene che l'uomo può solo applicarsi a conoscere il piano di Dio, senza pretendere però di cambiarlo" (DI PALMA, G., Il giudizio di Dio nel libro di Qohelet, p. 363).

${ }^{45}$ FESTORAZZI, F., Il Qohelet, p. 188-189. A questo proposito, però, Festorazzi non entra nel dettaglio della problematica, presentando l'esigenza di "un rapporto diverso con un Dio diverso da quello tradizionale" come qualcosa che sorge non da un testo specifico ma dalla visione globale della realtà espressa da Qohelet. La questione meriterebbe comunque di essere approfondita ulteriormente e discussa. 
maestro palestinese sa di Dio, gli proviene dal dogma della fede d'Israele; ma ciò che egli osserva nella vita quotidiana è costituito da tutti i fenomeni che si realizzano sotto il sole e che raccontano una storia diversa, ossia la storia del mondo come appare all'osservatore. Il dogma narra invece la storia del mondo, come essa è creduta dal pio israelita. Sono questi i due poli da non confondere nella nostra esegesi del libro di Qohelet. Possiamo quindi affermare, con F. Festorazzi, che il timore di Dio implichi un rapporto di sottomissione al Dio radicalmente trascendente e incomprensibile, e richieda, al contempo, anche una fiducia di base nella bontà del suo governo universale. Ma questo Dio non ci sembra diverso da quello tradizionale. Almeno non lo è sul piano della fede d'Israele. Tuttavia, osservando la sua regia quotidiana, molte cose che accadono nel mondo, potranno risultare incomprensibili alla mente umana. ${ }^{46}$ Per Qohelet, questo Dio rimane sempre e comunque creatore e origine della vita, agendo nel mondo come giudice e rimuneratore, arbitro dei destini umani, sorgente della gioia, termine ultimo del pellegrinaggio terreno dell'uomo.

Queste disposizioni d'animo delineate circa il timore di Dio come virtù, valgono a descrivere quel sapiente che abbiamo distinto dalla persona semplicemente giusta. Si dà, però, un ulteriore ambito di manifestazione del timore di Dio: quello del culto. La pericope di riferimento è Qo 4,17-5,6. ${ }^{47} \mathrm{Ne}$ diamo qui la nostra traduzione:

\begin{abstract}
Osserva i tuoi passi quando vai verso la casa di Dio: avvicinarsi per ascoltare è più dell'offerta sacrificale degli stolti, che non comprendono di compiere un male. Non essere precipitoso con le labbra e il tuo cuore non si affretti a pronunciare una parola al cospetto di Dio, perché Dio è in cielo e tu sei sulla terra. Quindi, le tue parole siano contate. Il sogno proviene da molte preoccupazioni e la voce dello stolto da molte parole. Così, una volta pronunciato un voto, non differire il suo compimento. Infatti, negli stolti
\end{abstract}

\footnotetext{
${ }^{46}$ L. Mazzinghi ha osservato che l'enfasi posta da Qohelet sulla divina trascendenza sottolinea la libertà di Dio, che agisce come un sovrano, le cui decisioni possono sembrare arbitrarie alla mente umana, ma non è necessariamente così a priori (MAZZINGHI, L., The Divine Violence in the Book of Qoheleth, p. 554). Appunto: che Dio governi il mondo come un bravo sovrano è un dogma; che la mente umana talvolta possa giudicare arbitrari i suoi decreti, è invece il risultato della semplice osservazione empirica.

${ }^{47}$ Non occorrono troppe dimostrazioni per delimitare la pericope: essa è chiaramente determinata da un brusco cambiamento tematico in 4,17 , passando dalla narrativa didattica alla forma esortativa in seconda persona. In 5,7, poi, si ha un ulteriore cambiamento: dal tema del timore di Dio a quello della giustizia sociale.
} 
non c'è zelo; pertanto, ciò che intendi promettere, adempilo. È meglio non promettere affatto, piuttosto che fare un voto e non mantenerlo. Non permettere alla tua bocca di condurti verso il peccato. Non pronuncerai una parola dinanzi al messaggero, ${ }^{48}$ per poi dire che è stata una sbadataggine; perché Dio dovrebbe sdegnarsi per la tua frase e distruggere l'opera della tue mani? ${ }^{49}$ Infatti, nei molti sogni c'è inconsistenza, insieme a molte parole. Abbi, dunque, timore di Dio.

Questo passaggio ci sembra di estrema importanza, in quanto dimostra, senza possibilità di equivoci, che Qohelet è sostanzialmente un pio Israelita e non uno scettico razionalista. Sembra del tutto pertinente, a questo riguardo, l'osservazione di J. V. Líndez: "Per lui l'autentico culto a Dio è una cosa molto seria, data la sua profonda concezione della trascendenza e, di conseguenza, della vera religiosità umana". ${ }^{50}$ Il culto, analogamente ai sentieri della sapienza, non può essere autentico senza il timore di Dio. La critica della religiosità, elaborata dal maestro, ingloba chiaramente le istanze del profetismo, ${ }^{51}$ nel momento in cui egli proclama il primato dell'ascolto sul gesto rituale, che rischia di mutarsi in un procedimento meccanico e senz'anima. ${ }^{52}$ Ciò risulterebbe logicamente offensivo per la divina maestà.$^{53}$ Ma riecheggia pure gli insegnamenti della tradizione sapienziale. In definitiva,

48 Traduco con "messaggero" il termine מלאך evitando di trasformarlo in אלהים come viene suggerito dalle versioni della LXX e della Siriaca. L'appellativo di "messaggero" qui può riferirsi plausibilmente a un sacerdote del tempio, che il pio israelita trova come proprio interlocutore in materia di voti e di sacrifici rituali.

${ }^{49}$ Abbiamo preferito leggere la particella למה in senso interrogativo e non finale, come fanno taluni (MURPHY, R., Ecclesiastes, p. 44; SEOW, C. L., Ecclesiastes, p. 193; LÍNDEZ, J. V., Qoèlet, p. 282).

${ }^{50}$ LÍNDEZ, J. V., Qoèlet, p. 281

51 "Thus its relativization of the value of sacrifice through 'hearing' corresponds to prophetic traditions and their 'Deuteronomic' reception, and it fits in perhaps with contemporary piety movements criticizing temple and sacrifice" (KRÜGER, T., Qohelet, p. 111).

${ }^{52} \mathrm{Si}$ può ricordare, a questo proposito, la condanna di un culto sganciato dall'agire morale, chiaramente espressa dai profeti preesilici: Is 1,10-17; Os 6,5-6; Am 5,21-24. Ma anche dopo, quando il tempio viene ricostruito al ritorno dell'esilio, la tematica non cambia: il profeta Malachia rivolge ai sacerdoti un chiaro rimprovero: il sacrificio rituale non è accettabile, se non si coniuga con l'agire morale e con il rispetto dovuto alla santità di Dio (M1 1,10-14).

${ }^{53}$ Il profeta Malachia esprime con fine umorismo quale sia il valore di un animale difettoso sacrificato a Dio, risparmiando astutamente quello sano. Egli invita i sacerdoti del tempio a offrire in dono un animale zoppo o malato al governatore, osservando poi la sua reazione (Ml 1,8). 
l'antidoto a questa forma di patologia del culto è individuato da Qohelet in tre suggerimenti basilari, provenienti dal cuore dell'etica biblica: il primato dell'ascolto, il controllo del linguaggio e la fedeltà al voto fatto. È di chiara matrice sapienziale l'invito a non avere fretta a pronunciare parole davanti a Dio (Qo 5,1-2). La fretta è, infatti, considerata una cattiva consigliera dalle tradizioni di epoca monarchica (Prv 19,2) e le molte parole sono giudicate come il marchio distintivo del discorso dello stolto (Prv 10,19). Se gli stolti non possono offrire alcun sacrificio gradito a Dio (Qo 4,17), allora l'uso sobrio del linguaggio non può che essere parte integrante di una corretta religiosità. Nella tradizione sapienziale si registra, in rapporto alla preghiera del saggio, una preferenza della preghiera semplice e non eccessivamente verbosa. Per il libro del Siracide, l'accumulo di parole va evitato in due precisi contesti, quello dell'assemblea degli anziani e quello della preghiera (Sir 7,14). Potrebbe pure darsi che in questa prospettiva possa convergere la tradizione sacerdotale, come qualcuno ha suggerito, sotto l'aspetto di uno stile da tenersi nel luogo sacro, dove si esige la pratica del silenzio. ${ }^{54}$ È possibile. Ad ogni modo, dal nostro punto di vista, sarebbe un'ulteriore conferma della pietas israelitica del maestro palestinese. L'invito a tacere davanti a Dio non è, comunque, un concetto estraneo neppure al profetismo preesilico; basti pensare ad Abacuc $(2,20)$ e a Sofonia $(1,7)$.

L'affermazione sulla trascendenza di Dio, secondo cui "Dio è in cielo e tu sei sulla terra" (Qo 5,1c), entra in pieno, e coerentemente, nella teologia di Qohelet già messa precedentemente in luce. Diremmo, unitamente a William P. Brown, che "Dio è al di là o al di sopra del cuore e della mente, al di là dei sentimenti e dei pensieri, al di là delle aspettative e dei desideri umani". ${ }^{55}$ Non si insisterà abbastanza su questo punto: il Dio di Qohelet dimora in un'irraggiungibile trascendenza. Da questa visione teologica deriva il ridimensionamento della sapienza e una particolare tonalità del timore di Dio. Ma nulla di veramente nuovo rispetto alla tradizione sapienziale che lo precede. Inoltre, affermare che Dio sia "in cielo", non equivale a definirne la lontananza o l'assenza dal mondo. Anzi, quasi sorprendendo il suo lettore, Qohelet prospetta qui una possibile retribuzione terrena: "perché Dio dovrebbe sdegnarsi per la tua frase e distruggere l'opera della tue mani?" (Qo 5,5). L'immagine è antropomorfica, ma intende esprimere

\footnotetext{
${ }^{54}$ BROWN, W. P., Qohelet, p. 74.

${ }^{55}$ BROWN, W. P., Qohelet, p. 75.
} 
un concetto molto chiaro, che il maestro desume non dalla vita quotidiana, ma dal dogma del Dio rimuneratore. Quando, invece, applica un metodo empirico di osservazione, e considera le dinamiche della vita reale, egli è costretto a formulare un giudizio ben diverso: non si dà sentenza immediata per ogni singola colpa e per questa ragione si accresce, nel cuore umano, il desiderio di agire empiamente (Qo 8,11).

Quanto all'adempimento di un voto fatto (Qo 5,3), la prospettiva presentata da Qohelet coincide, nelle linee essenziali, con esigenze espresse dalla Torah. La consuetudine di pronunciare dei voti è un tema toccato dalla legislazione mosaica sia nel libro dei Numeri sia nel Deuteronomio. In modo più specifico, quest'ultimo stabilisce che tra il pronunciamento del voto e il suo adempimento non deve trascorrere un tempo troppo lungo, perché il ritardo è già una colpa di cui Dio chiederebbe conto all'orante (Dt 23,22). Il testo di Numeri, invece, stabilisce soltanto l'obbligo di adempimento, dopo l'atto di pronunciamento del voto, e ciò senza precisazioni temporali né previsione di sanzioni ( $\mathrm{Nm} 30,2)$. Qohelet ha presenti entrambe le normative, ma ha presente anche il dettato di Prv 20,25: "è una trappola per l'uomo affrettarsi a dire "sacro!" e poi riflettere a voti fatti". ${ }^{56}$ In sostanza, mentre la legge mosaica istituisce e regolamenta la pratica dei voti, la sapienza ne suggerisce un uso circospetto, data la gravità della materia. Il maestro si spinge perfino oltre, radicalizzando l'insegnamento sapienziale, e superando le consuetudini religiose d'Israele, fino a consigliare ai suoi discepoli di non fare voti di alcun genere, cosa assai migliore rispetto al pericolo, sempre possibile, dell'inadempienza. Il versetto conclusivo della pericope, invitando al timore di Dio, sembra sintetizzare in esso il triplice antidoto alla possibile degenerazione del culto: 1'ascolto ubbidiente, la prudenza della parola e la consapevolezza della gravità dei voti pronunciati davanti a Dio. ${ }^{57}$

\subsection{Una possibile escatologia in Qohelet?}

Prima di affrontare un tema così delicato, quale quello della speranza, occorre precisare che cosa sia l'escatologia e cosa debba intendersi nel

\footnotetext{
${ }^{56}$ La traduzione italiana è nostra.

${ }^{57}$ Accogliamo senza riserve quanto osserva C.-L. Seow: "Qohelet then concludes with an admonition to fear God, that is, respect the distance between the divine and the humane. This conclusion in fact summarizes the content of the entire passage" (SEOW, C. L., Ecclesiastes, p. 201).
} 
linguaggio teologico con una tale definizione. A questo scopo, vogliamo fare nostra la tesi di K. Rahner: "nella fede cristiana e nella sua asserzione ci deve essere una escatologia che intenda veramente la realtà futura, ciò che in un senso molto comune ed empirico non è temporalmente subentrato". ${ }^{58}$ Si può aggiungere che quanto viene detto della fede cristiana può essere esteso alla fede biblica, in quanto essa rappresenta, nel suo complesso, una religione della promessa. In questo caso, ciò che "non è temporalmente subentrato", è oggetto di speranza in due modi: il novum intrastorico, proveniente dall'annuncio profetico e radicalmente negato da Qohelet, e il novum ultraterreno, su cui occorre soffermarsi alquanto, perché la soluzione tradizionale dello sheol non spiega interamente la problematica del futuro, così come il maestro la affronta. Anche su questo settore si registrano i due livelli già menzionati, che spingono lo sviluppo del suo pensiero in due direzioni diverse e coesistenti. Soffermiamoci sui singoli passaggi.

Il primo testo che entra in merito al destino dell'essere umano dopo la morte è Qo 3,16-21. Ne diamo la nostra traduzione:

Ancora ho visto sotto il sole in luogo della giustizia, l'empietà; e in luogo dell'equità, l'ingiustizia. Dissi in cuor mio: il giusto e l'empio Dio giudicherà, poiché c'è un tempo per ogni affare e per ogni opera. Dissi in cuor mio a proposito degli uomini: Dio li passa al setaccio, ${ }^{59}$ per far vedere $^{60}$ loro che essi sono come bestie, poiché la sorte degli uomini e la sorte delle bestie è una sola; come muoiono questi, muoiono quelle e non c'è un di più per l'uomo, perché tutto è un soffio. Tutti vanno verso la stessa destinazione. Tutti i viventi vengono dalla polvere e tutti tornano nella polvere. Chi sa se lo spirito dei figli dell'uomo vada verso l'alto e quello della bestia scenda sotto terra?

L'indole empirica di questo brano è indubitabile. Un verbo di percezione (ראיתי) avvia l'intera pericope, che ha l'apparenza di un soliloquio. L'affermazione del giudizio di Dio su giusti ed empi in Qo 3,17 (ma anche l'esortazione individuale di Qo 11,9) non fa parte della sua indagine empirica

\footnotetext{
${ }^{58}$ RAHNER, K., Principi teologici dell'ermeneutica di asserzioni escatologiche, p. 399-440.

${ }^{59}$ Intendo il verbo ברר nel suo significato basilare di "separare" (tradotto dalla LXX con

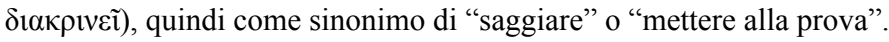

${ }^{60}$ Accettiamo la proposta dell'apparato critico di BHS che, sulla base della Siriaca e della Vulgata, cambia la forma infinitiva לראות in un infinito hiphil.
} 
ma della sua fede, ${ }^{61}$ mentre la prevalenza dell'empietà sulla giustizia risulta dalla sua osservazione dei fenomeni sociali. Dall'altro lato, l'uguaglianza di destino tra uomini e bestie è il frutto della semplice osservazione del meccanismo della vita. Agli occhi dell'osservatore esterno, infatti, la cessazione della vita biologica non manifesta sostanziali differenze tra la morte di un essere umano e quella di un qualsiasi animale. Chiunque avrebbe ragione, nell'affermare che "come muoiono questi, muoiono quelle e non c'è un di più per l'uomo" (Qo 3,19). Il fenomeno fisico del morire effettivamente non differisce. Questa constatazione, però, non equivale ad un'intenzionale negazione della sopravvivenza umana dopo la morte. ${ }^{62}$ Intendiamo dire che la precedente osservazione, circa la prevalenza dell'ingiustizia sull'equità (Qo 3,16 ), non smentisce il dogma del Dio giusto e Signore della storia, creduto da Qohelet e da ogni fedele Israelita. Sono semplicemente due grandezze giustapposte: Dio è giudice giusto (oggetto di fede); la vita sociale ha stravolto la giustizia (oggetto di esperienza). Al v. 20 il dogma e l'osservazione empirica s'incontrano: che tutti i viventi vengano dalla polvere non può essere osservato: si tratta di un'applicazione di Gen 2,7.19; 3,19b; il loro ritorno alla polvere, invece, appartiene a entrambi i livelli: l'enunciato riprende il testo di Gen 3,19d, ma costituisce anche una possibile osservazione connessa al seppellimento del cadavere. In definitiva, l'esistenza di questi due livelli contemporanei permette di leggere gli enunciati di Qohelet, senza alcuna problematica di ordine teologico; anzi, l'orizzonte dogmatico risulta quello tradizionale, mentre l'osservazione empirica mette in luce ciò che ciascun altro osservatore, in ciascun'altra generazione o epoca, avrebbe visto al posto

\footnotetext{
${ }^{61}$ È stato ampiamente dibattuto il problema relativo alla natura di questo giudizio di Dio, se si tratti cioè di un giudizio storico o escatologico. A nostro modo di vedere, la soluzione è semplice e non richiede grandi approfondimenti. Questa affermazione è soltanto l'espressione di un dogma di fede, che J. V. Líndez verbalizza con lucidità: "Per il credente, Dio è il Signore della storia, nella quale esercita realmente il suo potere totale sulla vita di ogni uomo [...]. Il giudizio di Dio non è oggetto di alcuna osservazione empirica, ma è il contenuto di un'affermazione di fede" (LÍNDEZ, J. V., Qoèlet, p. 258). Non ci sembra che occorra dire altro.

${ }^{62}$ Così infatti ritiene J. V. Líndez: "tanta insistenza nel parlare dello stesso destino dell'uomo e dell'animale non può avere che un unico significato: la negazione della sopravvivenza umana al di là della morte" (LÍNDEZ, J. V., Qoèlet, p. 262). Anche C.-L. Seow afferma che "Qohelet refuses to entertain any notion of separate destinies for the life-breaths of people and animals" (SEOW, C. L., Ecclesiastes, p. 176). T. Longman III preferisce non sbilanciarsi, sottolineando l'aspetto fenomenico di quanto accade agli esseri viventi nel loro morire e affermando che, in fondo, "it is difficult to know what the common theological belief about the afterlife was at the time of this writing" (LONGMAN III, T., The Book of Ecclesiastes, p. 129).
} 
suo. Inoltre, che Dio metta alla prova l'uomo, passandolo "al setaccio" (Qo 3,18 ), per condurlo come un pedagogo a una più lucida coscienza di sé, non fa parte di una constatazione oggettiva, né può essere empiricamente dimostrato, ma è un'idea desunta dalla torah e dalla tradizione sapienziale. ${ }^{63}$ Ciò fa quindi parte del creduto. Qohelet accetta questa interpretazione come vera e semplicemente la applica ai sentimenti di chi ha sotto gli occhi il fenomeno fisico del morire: questi impara ad accettarsi come creatura, se non fugge dal lutto come fanno gli stolti (Qo 7,2). Per lui è, insomma, di estrema importanza il ridimensionamento della volontà di potenza, che è l'atteggiamento esattamente opposto a quello della creaturalità. In un'altra occasione ho osservato che la riflessione sulla morte, come Qohelet la concepisce, è una tappa imprescindibile per demolire le illusioni derivanti dalla gloria umana e dal potere politico ed economico. ${ }^{64}$ Significativamente, i sovrani ellenistici pretendevano onori divini. Ciò dimostra che non è difficile assolutizzare certi valori per natura relativi, smarrendo il realismo della sana ragione. Per il maestro, chi riflette sulla fine dell'essere umano, non cade in queste derive intellettuali e pratiche. La casa in lutto è, infatti, offerta ai suoi discepoli come un efficace antidoto (Qo 7,2).

Particolare attenzione merita, in rapporto alla teologia della speranza, la domanda posta dal maestro in 3,21: "Chi sa se lo spirito dei figli dell'uomo vada verso l'alto e quello della bestia scenda sotto terra?". Questo interrogativo si discosta sia dalla concezione tradizionale dello sheol, ${ }^{65}$ sia dall'osservazione empirica. Quest'ultima, infatti, non ha alcun legame con il possibile viaggio ultraterreno della רוח dopo la cessazione della vita fisica. Ma anche l'ipotesi di un movimento verso "l'alto" non è sufficientemente suffragata dalla tradizione. Ciò esige un'ulteriore indagine. Fa problema innanzitutto il termine רוח che si riferisce principalmente al respiro. Taluni esegeti concludono, quindi, che non si tratta dell'io cosciente che può sussistere dopo la morte. J. V. Líndez sintetizza così il risultato della ricerca recente: "secondo l'unanime testimonianza degli autori moderni il termine ruah 'non è ciò che nella nostra concezione sopravvive alla morte del corpo

\footnotetext{
${ }_{63}$ Possiamo opportunamente ricordare, a questo proposito, che la sofferenza d'Israele nel deserto è concepita in senso pedagogico dal redattore di Dt 8,3-5, mentre la sapienza assume i caratteri di una severa disciplina in Sir 2,4-5 e 4,17-18.

${ }^{64}$ CUFFARO, V., Il problema della morte in Qoelet, p. 130-131.

${ }^{65}$ Qohelet espone la concezione tradizionale dello sheol in 9,3-6.10: esso è il luogo dell'oblio e della non conoscenza; i morti non vi hanno alcun salario e anche i loro sentimenti risultano annullati. L'unica fase positiva dell'esistenza è la vita terrena.
} 
umano', ma alito, soffio vitale, principio di vita". ${ }^{66}$ Siamo d'accordo: $r u a h$ non è l'anima umana come la teologia scolastica l'ha intesa. Ma è davvero solo un soffio che si disperde o in alto o in basso? Nel libro di Giobbe, la ruah umana è descritta nell'atto di tornare a Dio, il che - a nostro giudizio - non può implicare un suo dissolvimento nel nulla: "Se [Dio] stabilisse in cuor suo di richiamare il suo respiro (רוח) e il suo soffio vitale (נשמה), morirebbero i viventi simultaneamente e l'uomo tornerebbe alla polvere (עפר)" (Gb 34,14). ${ }^{67}$ Il poeta dà qui una precisa interpretazione della morte come l'inizio di un duplice viaggio: il corpo e la ruah (cioè la dimensione interiore e invisibile) nell'atto di separarsi, imboccano due direzioni diverse. Qohelet conosceva questo testo e qui lo ha richiamato indirettamente, insieme a quelli della Genesi, che si percepiscono tra le righe: mentre la ruah torna a Dio, il corpo fisico torna alla terra.

La questione relativa alla ruah umana, torna in un secondo testo dedicato a questo tema; esso mostra esattamente la medesima dinamica rinvenuta nella pericope già analizzata. Si tratta di Qo 12,7: “Torni la polvere (עפר) alla terra, come era prima, e il respiro (רוח) a Dio che l'aveva donato". Combinando i due testi biblici sul tema della morte, Gen 2,7; 3,19 e Gb 34,14, ne emerge una speranza embrionale sul destino ultraterreno dell'essere umano come possibile ritorno a Dio della ruah, ${ }^{68}$ dal momento che Lui ne è il donatore e dunque anche il proprietario. Che il termine ruah per l'essere umano possa rappresentare non solo il meccanismo fisico del respiro, ma qualcosa di più sul piano psicologico, risulta proprio dalla sua destinazione אל־האלהים. E risulta anche dal fatto che i termini antropologici usati dall'AT possiedono un carattere piuttosto elastico: essi non indicano soltanto l'elemento che direttamente descrivono, ma hanno anche la capacità di indicare la persona in modo generico. Così termini come בשר ,נשמה, possono indicare - come si evince dai dizionari scientifici - rispettivamente il respiro, lo stato d'animo e la sfera dell'interiorità; il soffio vitale, l'essere vivente e l'alito; il corpo fisico e la persona. Il primo di questi termini antropologici, che riguarda direttamente

\footnotetext{
${ }^{66}$ LÍNDEZ, J. V., Qoèlet, p. 264-265.

${ }^{67} \mathrm{La}$ traduzione dall'ebraico è nostra.

${ }^{68} \mathrm{~T}$. Krüger si mostra dell'idea che la separazione tra corpo e ruah non sia oggetto di speranza ma indichi piuttosto “the definitive end" dell'essere umano (KRÜGER, T., Qohelet, p. 203). Anche T. Longman III si schiera nella linea negativa: "This is not an optimistic allusion to some kind of consciousness after death, but simply a return to prelife situation" (LONGMAN III, T., The Book of Ecclesiastes, p. 273), insieme a R. Murphy: "This is a picture of dissolution, not of immortality, as if there were a reditus animae ad Deum" (MURPHY, R., Ecclesiastes, p.120).
} 
la nostra indagine, se anche non descrive l'anima razionale nel senso inteso dalla teologia scolastica, nondimeno indica, in Qo 12,7 , l'interiorità umana, ${ }^{69}$ descritta nel suo possibile ritorno a Dio dopo la morte del corpo. ${ }^{70} \mathrm{La}$ domanda che il maestro aveva posto in Qo 3,21 proveniva dalla sfera dell'osservazione empirica, nella quale non si può dire nulla circa la destinazione dell'uomo o della bestia, dopo la loro morte. Tuttavia, egli non vuole lasciare l'interrogativo senza risposta e, non potendo risolverlo sul piano empirico, attinge a quello dogmatico, trovando due brani biblici pertinenti e utili allo scopo: il secondo racconto della creazione e il testo, già citato, del libro di Giobbe.

\section{Il Dio di Qohelet e l'uomo contemporaneo}

Coloro che credono in un solo Dio, e in particolare gli ebrei e i cristiani, si trovano dinanzi al mondo e alla storia recente in una posizione di maggiore crisi rispetto a quella del saggio Qohelet. Essi hanno conosciuto due guerre mondiali. Gli scenari dei bombardamenti, con le rovine di grandi città rase al suolo, insieme all'orrore dei campi di concentramento, sono ancora parte integrante dell'immaginario collettivo, anche delle generazioni che non ne sono state contemporanee. Il periodo postbellico, però, non ha condotto a una vera pace, bensì a una nuova forma di guerra, definita "fredda", che ha continuato a dividere il mondo occidentale in due blocchi fino al suo termine, avvenuto con la caduta del muro di Berlino nel 1989 e con la successiva dissoluzione dell'URSS (1991). Nonostante la fine dei grandi conflitti, dopo gli anni Sessanta, nel mondo si registra ancora un altro grave fenomeno: una serie di massacri e genocidi, che segnano la storia del secolo XX e toccano ampi settori del pianeta, quali l'Indonesia, la Cambogia, l'Africa, l'America latina, il medio Oriente, l'Europa dell'est. Ma questo non è ancora tutto: ai mali del passato, si aggiungono anche alcuni timori non piccoli, connessi

\footnotetext{
${ }^{69}$ BROWN, F.; DRIVER, S. R.; BRIGGS, C. A., רוח, p. 925, ad vocem: “3. spirit as that breathes quickly in animation or agitation = temper, disposition"; KOEHLER, L.; BAUMGARTNER, W., רוח, p. 878, ad vocem: "6. Geist des Menschen; 7. Sinn, Gesinnung, geistige Verfassung"; ALONSO SCHÖKEL, L., רוח, p. 774-775, ad vocem: "2. Alito vitale, anima, spirito; 3. Designa o significa la sfera dello spirito, della coscienza, dell'interiorità". Osserva H. W. Wolff che "Mit dem Wehen des Atems ist nämlich zunächst die Bewegung des Gemüts zusammenzusehen" (WOLFF, H. W., Anthropologie des Alten Testaments, p. 63).

${ }^{70} \mathrm{Mi}$ trovo in linea, in questa prospettiva escatologica del non dissolvimento della ruah umana dopo la morte, con la posizione di A. C. T. Nguyen Thi su Qo 12,7, espressa nel suo articolo: NGUYEN THI, A. C. T., La destinée de l'Homme chez Qohelet (Qo 1,4-11; 12,1-7), p. 236-237.
} 
alle minacce del presente: l'insorgere del terrorismo internazionale, che colpisce all'improvviso e fa strage di innocenti; gli armamenti nucleari, il cui possibile uso potrebbe mutare drammaticamente l'attuale assetto del mondo; il cambiamento climatico, che ci espone a fenomeni atmosferici estremi e non sempre controllabili; l'emergenza causata dal traffico di migranti e il costo di vite umane, che inevitabilmente ne consegue. Un teologo non può sottovalutare la gravità di questi eventi, che vanno confrontati con la rivelazione biblica. Ma nemmeno il credente in quanto tale, può fare a meno di interrogarsi.

Rimanendo però all'interno del nostro tema, dobbiamo dire che $\mathrm{i}$ paradossi osservati da Qohelet, le ingiustizie e le oppressioni da lui denunciate, sono retaggio perenne della storia umana, e anche la nostra generazione ne fa esperienza, con una non minore esigenza - rispetto a quelle già passate - di un orizzonte di senso. Sotto questo profilo, suonano opportune le espressioni del maestro, secondo cui le generazioni trascorrono ma la terra rimane e non c'è nulla di nuovo sotto il sole (Qo 1,2-11). Tuttavia, l'umanità contemporanea si trova dinanzi a sfide che la generazione di Qohelet non conosceva. Il potere di controllo, raggiunto oggi dall'umanità nei confronti del creato, non ha paragoni; di conseguenza, i paradossi da noi fronteggiati sono molto più gravi ed estremi di quelli che un tempo affaticarono la riflessione del saggio palestinese. E così, la nostra generazione si mostra a un tempo forte e debole, capace di operare il massimo bene e il massimo male. ${ }^{71}$

Nondimeno, Qohelet ha ancora qualcosa da dirci. Quanto è emerso nel corso della nostra ricerca, è già utile all'identificazione di alcuni orientamenti da offrirsi al lettore odierno. In primo luogo, citerei l'invito a ridimensionare la volontà di potenza, che scaturisce dal progresso tecnologico. Egli non conosceva la tecnologia, ovviamente, ma le sue osservazioni sull'approccio psicologico del sapiente con la vita, vanno in questa direzione. Ci sembra significativo, a questo riguardo, l'esortazione rivolta all'esuberanza giovanile in Qo 12,1: "ricordati del tuo creatore nel tempo della tua giovinezza". L'età giovanile è, infatti, ignara del limite sperimentato dagli anziani, quando le energie vitali declinano. La cognizione del proprio essere mortale non è sufficientemente lucida in una persona che si trova ancora nel pieno possesso della sua salute e dei suoi anni, finché il futuro è ancora abbastanza più lungo del passato. ${ }^{72} \mathrm{Vi}$ sono tuttavia altre condizioni

${ }^{71}$ GS 9.

${ }^{72}$ A. C. T. Nguyen Thi ha individuato questo aspetto psicologico non trascurabile nell'esortazione di Qohelet al giovane, osservando che l'espressione di Qo 12,1 "suggère que la jeunesse risque 
che possono offuscare la propria creaturalità, come la possibilità tecnica di manipolazione della natura, insieme a tutte le altre forme del potere fisico e morale. L'invito a ricordarsi del proprio creatore risulta pertanto di estrema attualità in un mondo che il Concilio Vaticano II ha definito come capace di risultati estremi. ${ }^{73} \mathrm{~A}$ ciò si aggiunge la prospettiva, più volte sottolineata da Qohelet, di un'irriducibile distanza tra gli sforzi dell'indagine umana e le ragioni ultime delle cose, tanto da circoscrivere la validità della sapienza alle questioni pratiche e non definitive (Qo 7,23-24 e 8,17), mentre il dolore cresce in proporzione al sapere (Qo 1,18).

Inoltre, egli spinge il lettore credente a non ritenere che le contraddizioni della storia e la prevaricazione dell'ingiustizia dimostrino la debolezza di Dio, oppure la sua assenza reale dal mondo umano. Ciò si evince chiaramente dal fatto che il dogma non viene svilito dall'osservazione dell'esperienza quotidiana. Il Dio di Qohelet è creatore, legislatore e giudice dei destini umani. L'origine del male che travaglia la società non è attribuita a Dio, ma neppure a un'entità maligna esterna a Dio. ${ }^{74}$ Si è visto piuttosto che la sorgente del male morale è posta nell'esercizio improprio della libertà umana. Per quel che riguarda, poi, il male fisico in tutti i suoi aspetti: personale, ambientale e cosmico, Dio è chiamato in causa non come responsabile colpevole, ma come regista di un equilibrio incomprensibile tra ordine e caos. E non potrebbe essere diversamente, dal momento che il dogma del Dio creatore e giudice è condiviso da Qohelet e costituito come base della sua ricerca sapienziale. Ma anche nell'apparente prevalenza del caos, per chi - come lui - ha accolto il dogma biblico, l'assenza di Dio non è un fatto reale, ma solo l'effetto della percezione umana. ${ }^{75}$ Infatti, la chiave di comprensione della realtà, in senso globale, non è in possesso del saggio. In ogni caso, seguendo i risultati della ricerca empirica di Qohelet, in merito al problema del male, si può cogliere un orientamento che verbalizzerei così: se proprio si vuole avviare una riflessione sull'origine dei guai che colpiscno la nostra società, non

d'occulter, chez l'homme, la conscience d'être une créature mortelle" (NGUYEN THI, A. C. T., La destinée de l'Homme chez Qohelet (Qo 1,4-11; 12,1-7), p. 231).

${ }^{73}$ GS 9.

${ }^{74}$ CARNY, P., Theodicy in the Book of Qohelet, p. 80.

${ }^{75}$ Condividiamo qui l'osservazione conclusiva di L. Mazzinghi, secondo cui Qohelet non descrive il suo Dio come un assente, o un ingiusto o un violento, ma semplicemente aldilà di ogni possibilità di comprensione (CARNY, P., Theodicy in the Book of Qohelet, p. 558). 
bisogna chiamare in causa il governo di Dio, ma l'esercizio scorretto della nostra libertà.

Infine, non è di secondaria importanza, nel pensiero di Qohelet, il tema del momento presente. La felicità non va cercata in grandi o imponenti realizzazioni, ma nella capacità di vivere ogni istante con intensità. Il maestro allude spesso al mangiare e al bere come ad atti di una quotidianità apparentemente scontata, ma ricca di significato per chi sa viverla senza banalizzarla. I grandi progetti e le grandi realizzazioni della vita sono perlopiù oggetto di delusione e vengono, perciò, raccolti globalmente sotto la categoria di inconsistente soffio (הבל). Ciò che rimane alla persona come effettiva esperienza positiva è il momento presente, in quanto in esso può fruirsi una gioia donata da Dio. Sotto questo profilo, ci sembra plausibile la duplice distinzione operata da M. Maussion, secondo cui in Qohelet esistono due tipi di gioia: quella derivante dal successo e quella donata da Dio. ${ }^{76}$ Ebbene, la prima delle due è altisonante ma inconsistente; la seconda è invece capace, senza fare rumore, di riempire l'animo umano di un complessivo e sostanziale benessere. ${ }^{77}$ Una filosofia di vita di questo genere, possiede senz'altro una validità senza tempo.

\section{Conclusione}

Prima di congedarci, è opportuno riprendere, nelle linee essenziali, quanto emerso dalla presente ricerca. Innanzitutto dobbiamo osservare che molti equivoci si sono addensati sull'opera di Qohelet nell'arco di questi ultimi cinquant'anni. Non di rado giudizi opposti sono stati formulati dagli studiosi. Ciò è indicativo, senza dubbio, della ricchezza insita in questo piccolo libro, che tuttavia ha ancora bisogno di essere compreso nella sua giusta luce. Ci sentiamo di affermare con W. Zimmerli che il Dio di Qohelet è il Dio della tradizione giudaica. Egli è creatore e custode

\footnotetext{
${ }^{76}$ MAUSSION, M., Qohélet VI 1-2, p. 507.

${ }^{77}$ Qohelet attinge al dogma per indicare quale sia l'alternativa sicura alle delusioni dei grandi progetti della vita, ossia il gusto del momento presente: "la sua fede ebraica lo porta a riconoscere questa possibilità come un dono del Creatore; egli utilizza il termine tob, per definire le gioie della vita quotidiana, con chiaro riferimento al giudizio che accompagna l'opera della creazione, secondo il racconto Sacerdotale. Tutto quello che Dio ha creato è buono. È quindi orientato alla consolazione dell'uomo, che ha un difficile compito sulla terra e che porta avanti le sue opere con fatica, sperimentando contro di sé la corrosione del tempo e la forza distruttiva del male" (CUFFARO, V., Epicuro e Qoelet, p. 283-284).
} 
della vita e arbitro dei destini umani. Questa visione di Dio è tratta dalla rivelazione biblica e non è in alcun modo deducibile dall'osservazione dell'esperienza. L'indagine empirica, invece, che caratterizza il metodo base del lavoro dei saggi d'Israele, si trova dinanzi a uno scenario del tutto diverso. Dall'incompatibilità di questi due piani nasce il doppio binario della riflessione del maestro e non, come ritiene M. V. Fox, dal confronto tra la storia e le esigenze dell'umano raziocinio. ${ }^{78} \mathrm{La}$ ragione umana, senza il supporto del dogma, non coglie alcuna assurdità nei fenomeni della natura e della storia, come lo dimostra il fatto che tutto appare ben ordinato ai filosofi precristiani, perfino ad atomisti come Leucippo e Democrito, comunemente ricordati come chi "il mondo a caso pone". ${ }^{79} \mathrm{La}$ ribellione della ragione al disordine naturale o storico può nascere soltanto dalla promessa di un mondo regolato da un legislatore universale, contro il quale possono rivolgersi i sospetti e le accuse delle delusioni. Ebbene, ci sembra di poter collocare il pensiero di Qohelet al crocevia di questa problematica. Due istanze contribuiscono all'elaborazione del pensiero del maestro: la promessa di un mondo come entità morale, determinato dal concetto di creazione, e l'osservazione della vita attraverso il metodo empirico dei sapienti d'Israele. In altre parole, il dogma e l'esperienza, ovvero due elementi che narrano storie diverse, ma entrambe vere. Ciò spiega la vera natura di tutti gli enunciati apparentemente contraddittori: il dogma presuppone che Dio abbia fatto bene ogni cosa (Qo 3,11), mentre l'esperienza dimostra che vi sono delle cose storte non raddrizzabili (Qo 1,15). Il dogma afferma che la felicità è un dono di Dio (Qo 2,24), mentre l'esperienza mostra che molti soffrono senza il sollievo di un consolatore (Qo 4,1). Il dogma insegna che Dio retribuisce, giudica e distingue i destini del giusto e dell'empio (Qo 7,18; 11,9), ma l'esperienza racconta un'altra storia (Qo 9,2). La lista potrebbe continuare, nondimeno è sufficiente questo per comprendere come le contraddizioni del maestro palestinese siano solo apparenti, determinate cioè da due cognizioni diverse ma valide entrambe: la fede e l'esperienza della vita.

Una dimostrazione del dogma di Israele, assunto da Qohelet come presupposto della sua ricerca sapienziale, si coglie poi nella prospettiva

${ }^{78}$ FOX, M. V., The inner-structure of Qohelet's thought, pp. 225-238.

${ }^{79}$ Più precisamente il detto riguarda il solo Democrito; è Dante Alighieri che lo definisce così, incontrandolo nel limbo insieme ad altri filosofi antichi: "Democrito, che 'l mondo a caso pone / Dïogenès, Anassagora e Tale, / Empedoclès, Eraclito e Zenone” (Inferno IV,136-138). 
etica che attraversa il libro. Il timore di Dio e il libero arbitrio sembrano costituire la strada maestra del suo insegnamento morale. Più precisamente, il timore è una delle due vie che si biforcano dinanzi alla scelta libera del singolo individuo. La seconda è l'empietà. L'esercizio della libertà del volere è possibile solo a partire da un'opera compiuta da Dio (Qo 3,14). Non esiste, infatti, alcuna libertà laddove non ci sia qualcosa, dinanzi a cui prendere una decisione. La sua visione del culto, poi, si cala nella più tradizionale linea profetica e sapienziale, condannando il culto sganciato dalla giustizia sociale e mettendo in guardia i suoi discepoli contro ogni forma di frettolosità.

Infine, ci siamo interrogati sulla possibilità che in Qohelet possa riscontrarsi una qualche forma di speranza escatologica. La maggioranza degli studiosi è nettamente contraria a una tale prospettiva. Nondimeno, il secondo racconto della creazione e il testo di Gb 34,14, entrambi presenti - a nostro giudizio - nella riflessione del maestro palestinese sulla morte, contengono le basi per definire un duplice viaggio del corpo e della ruah umana, che ha inizio al momento del decesso. Il corpo torna alla polvere ma la ruah sale verso Dio. Che cosa Qohelet intenda per ruah è deducibile dall'area semantica abbracciata dal lemma stesso, come si è dimostrato: si tratta del respiro come meccanismo della vita, ma si tratta anche dell'interiorità umana come insieme delle disposizioni d'animo. Vale a dire, quella dimensione interiore nella quale la persona può conoscere e incontrare Dio. Certo non si tratta di una vera e propria speranza escatologica, ma di qualcosa che ne costituisce quantomeno il fondamento embrionale.

\section{Riferimenti bibliografici}

ALONSO SCHÖKEL, L. רוח. In: ALONSO SCHÖKEL, L. Dizionario di Ebraico Biblico. Consulenza scientifica di Gian Luigi Prato. Cinisello Balsamo: Edizioni San Paolo, 2013. p. 774-775.

BROWN, W. P. Qohelet. Torino: Claudiana, 2012.

BROWN, F.; DRIVER, S. R.; BRIGGS, C. A. רוח. In: BROWN, F.; DRIVER, S. R.; BRIGGS, C. A. A Hebrew and English Lexicon of The Old Testament. Oxford: Clarendon Press, 1906. p. 925.

CARNY, P. Theodicy in the Book of Qohelet. In: REVENTLOW, H. G.; HOFFMAN, Y. Justice and Righteousness. Biblical Themes and their 
influence. United Kingdom: Sheffield, 1992. p. 71-81. (Journal for the Study of the Old Testament Supplement Series, 137).

CONCILIO VATICANO II. Costituzione pastorale sulla Chiesa nel mondo contemporaneo Gaudium et Spes. Disponibile in: <http://www.vatican.va/ archive/hist_councils/ii_vatican_council/documents/vat-ii_const_19651207_ gaudium-et-spes_it.html >. Visitato in: 09 gen. 2020.

CUFFARO, V. Il problema della morte in Qoelet. Studia Moralia, v. 56, n. 1, p. 123-144, 2018.

CUFFARO, V. Epicuro e Qoelet: il piacere, il possesso e la morte. Studia Moralia, v. 51, n. 2, p. 273-296, 2013.

D'ALARIO, V. L'assurdità del male nella teodicea di Qohelet. In: FABRIS, R. (a cura di). Initium Sapientiae. Scritti in onore di Franco Festorazzi nel suo 70 compleanno. Bologna: EDB, 2000. p. 179-197.

DANTE, A. La Divina Commedia. Milano: Ulrico Hoepli, 1989.

DE JONG, S. God in the Book of Qohelet: a reappraisal of Qohelet's place in Old Testament theology. Vetus Testamentum, v. 47, n. 2, p. 154-67, 1997.

DI PALMA, G. Il giudizio di Dio nel libro di Qohelet. Asp, v. 40, p. 349-72, 1993.

FESTORAZZI, F. Il Qohelet: un sapiente d'Israele alla ricerca di Dio. Ragione e fede in rapporto dialettico. In: DANIELI, G. Quaerere Deum. Atti della XXV Settimana Biblica. Paideia: Brescia 1980. p. 173-190.

FOX, M. V. The inner-structure of Qohelet's thought. In: SCHOORS, A. (Ed.). Qohelet in the Context of Wisdom. Leuven: Peeters Publishers, 1998. p. 225-238. (Bibliotheca Ephemeridum Theologicarum Lovaniensium, 136).

GIRE, P. Qohelet: l'espérance mediante. Lumière et Vie, v. 41, p. 41-54, 1995.

KAISER, O. Die Botschaft des Buches Kohelet. Ephemerides Teologicae Lovaniensis, v. 71, n. 1, p. 48-70, 1995.

KOEHLER, L.; BAUMGARTNER, W. רוח. In: KOEHLER, L.; BAUMGARTNER, W. Lexicon in Veteris Testamenti Libros. Leiden: E. J. Brill, 1985. p. 878.

KRÜGER, T. Qohelet: a Commentary. Minneapolis: Hermeneia / Fortress Press, 2004. 
LAHUA, A. Kohelet. Biblischer Kommentar Altes Testament. NeukirchenVluyn: Neukirchener Verlag, 1978.

LÍNDEZ, J. V. Qoèlet. Roma: Borla, 1997.

LOHFINK, N. Qohelet. Brescia: Morcelliana, 1997.

LONGMAN III, T. The Book of Ecclesiastes. Michigan / Cambridge U. K.: Eerdmans Publishing Company / Grand Rapids, 1998. (The New International Commentary on the Old Testament).

MAUSSION, M. Qohélet VI 1-2: “Dieu ne permet pas...”. Vetus Testamentum, v. 55, n. 4, p. 501-510, 2005.

MAZZINGHI, L. The Divine Violence in the Book of Qoheleth. Biblica, v. 90, n. 4, p. 545-558, 2009.

MURPHY, R. Ecclesiastes. Dallas / Texas: Word Books Publisher, 1992.

NGUYEN THI, A. C. T. La destinée de l'Homme chez Qohelet (Qo 1,4-11; 12,1-7). Revue Biblique, v. 120, n. 2, p. 220-239, 2013.

PIOTTI, F. Percezione del "disordine" e "timore di Dio" in Qohelet (II). Bibbia e Oriente, v. 51, n. 2/3, p. 101-131, 2009.

RAHNER, K. Principi teologici dell'ermeneutica di asserzioni escatologiche. In: RAHNER, K. Saggi sui sacramenti e sull' escatologia. Roma: Edizioni Paoline, 1969. p. 399-440.

RINGGREN, H.; ZIMMERLI, W. Sprüche / Prediger. Göttingen: Vandenhoeck \& Ruprecht, 1980. (Das Alte Testament Deutsch, 16/1).

SEOW, C. L. Ecclesiastes: a New Translation with Introduction and Commentary. New York: Doubleday, 1985. (The Anchor Bible, 18C).

VONACH, A. Gottes Souvränität anerkennen. Zum verständnis der "Kanonformel". In: SCHOORS, A. (Ed.). Qohelet in the Context of Wisdom. Leuven: Peeters Publishers, 1998. p. 391-397. (Bibliotheca Ephemeridum Theologicarum Lovaniensium, 136).

WHYBRAY, R. N. Qoheleth as a theologian. In: SCHOORS, A. (Ed.). Qohelet in the Context of Wisdom. Leuven: Peeters Publishers, 1998. p. 239-265. (Bibliotheca Ephemeridum Theologicarum Lovaniensium, 136).

WHYBRAY, R. N. Qoheleth the Immortalist? In: GAMMIE, J. G.; PERDUE, L. (Eds.). Israelite Wisdom: Theological and Literary Essays in Honour of Samuel Terrien. Missoula: Scholars Press, 1978. p. 191-204. 
WOLFF, H. W. Anthropologie des Alten Testaments. München: Kaiser Verlag, 1990.

\section{Vincenzo Cuffaro}

Doutor em Teologia Biblica na Facoltà Teologica di Sicilia, Palermo Docente da Facoltà Teologica di Sicilia e do Studio Teologico "San Gregorio Agrigentino" Agrigento - Italia E-mail: cuffarovincenzo@virgilio.it Aprovado em: 05/05/20 